\title{
COMPUTATION OF GENERALIZED STRESS INTENSITY FACTORS FOR BONDED ELASTIC STRUCTURES
}

\author{
MARIUS BOCHNIAK ${ }^{1}$ AND ANNA-MARgARETE SÄNDIG ${ }^{1}$
}

\begin{abstract}
We consider coupled structures consisting of two different linear elastic materials bonded along an interface. The material discontinuities combined with geometrical peculiarities of the outer boundary lead to unbounded stresses. The mathematical analysis of the singular behaviour of the elastic fields, especially near points where the interface meets the outer boundary, can be performed by means of asymptotic expansions with respect to the distance from the geometrical and structural singularities. The coefficients in the asymptotics, which are called generalized stress intensity factors, play an important role in classical fracture criteria. In this paper we present several formulas for the generalized stress intensity factors for $2 \mathrm{D}$ and $3 \mathrm{D}$ coupled elastic structures. The formulas have the form of scalar products or convolution integrals of the given data or the unknown displacement field and the so-called weight functions, similar to Maz'ya/Plamenevsky functionals introduced in [19] for elliptic boundary value problems. The weight functions are non- energetic elastic fields, which admit a decomposition into a known singular part and a more regular one, which is computed by boundary element domain decomposition methods. Numerical experiments for two-dimensional problems illustrate the theoretical results.
\end{abstract}

AMS Subject Classification. 35J55, 35C20, 73B50, 73K20, 73V10.

Received: September 12, 1997. Revised: May 25, 1998.

\section{INTRODUCTION}

Since the papers by Williams [35], Karp and Karal [14], and many others it is widely known that linear elastic and harmonic fields can have unbounded stresses in the neighbourhood of corners and edges of the boundary or near points where the boundary conditions change. Similar stress singularities can occur at points where the interface meets the outer boundary or at the front of a crack between dissimilar materials [36]. The singular behaviour of such fields can be described by means of asymptotic expansions with respect to the distance to the geometrical and structural singularities. If we denote by $(r, \omega)$ the local polar coordinates with origin in an interface corner point, then the asymptotics of the two-dimensional displacement or harmonic fields $u_{1}, u_{2}$ in the subdomains $\Omega_{1}, \Omega_{2}$ read as

$$
\left(\begin{array}{l}
u_{1} \\
u_{2}
\end{array}\right)(r, \omega) \sim \sum_{j} c_{j} r^{\alpha_{j}}\left(\begin{array}{l}
\varphi_{1, j}(\log r, \omega) \\
\varphi_{2, j}(\log r, \omega)
\end{array}\right)
$$

Keywords and phrases. Stress singularities, stress intensity factors, boundary element methods, error estimates, Aubin-Nitsche trick.

${ }^{1}$ Universität Stuttgart, Mathematisches Institut A, Pfaffenwaldring 57, 70569 Stuttgart, Germany.

e-mail: bochniak@mathematik.uni-stuttgart.de 
Asymptotic expansions of this type were derived by Kondrat'ev [15] for general elliptic boundary value problems using Mellin transformation techniques and were adapted to boundary transmission problems by Nicaise and Sändig [24]. For three-dimensional elastic displacement and harmonic fields near smooth edges or crack fronts the asymptotics can be expressed in cylindrical coordinates $(r, \omega, z)$

$$
\eta\left(\begin{array}{l}
u_{1}(r, \omega, z) \\
u_{2}(r, \omega, z)
\end{array}\right) \sim \eta \sum_{j} c_{j}(z) r^{\alpha_{j}(z)}\left(\begin{array}{l}
\varphi_{1, j}(\log r, \omega) \\
\varphi_{2, j}(\log r, \omega)
\end{array}\right),
$$

where the $z$-axis is tangential to the edge and $\eta$ denotes an appropriate cut-off function. Asymptotics of this kind were obtained by Maz'ya and Rossmann [20] for the Dirichlet problem in a bounded domain and for the Neumann and the mixed problem by Nazarov, Plamenevsky [22], Costabel and Dauge [6], Rossmann and Sändig [28].

In the classical two-dimensional linear fracture mechanics (see e.g. [13]) the leading term in the asymptotics of the displacement field near the tip of a crack in a homogeneous material is given in the form

$$
u(x) \sim \frac{K_{I}}{\mu}\left(\frac{r}{2 \pi}\right)^{1 / 2} f_{1}(\varphi)+\frac{K_{I I}}{\mu}\left(\frac{r}{2 \pi}\right)^{1 / 2} f_{2}(\varphi)+O(r),
$$

where $\mu$ is a material constant. The coefficients $K_{I}, K_{I I}$ are called stress intensity factors of opening and sliding mode, respectively. Their importance originates from the Irwin-Griffiths criterion for crack initiation in homogeneous materials [13]. A fracture criterion for the delamination of composite laminates has been proposed in $[12]$ in the form

$$
\left(\frac{K_{I}}{K_{I C}}\right)^{2.5}+\left(\frac{K_{I I}}{K_{I I C}}\right)^{3}+\left(\frac{K_{I I I}}{K_{I I I C}}\right)^{3}=1
$$

where $K_{I I I}$ denotes the stress intensity factor of the anti-plane deformation and the constants $K_{I C}, K_{I I C}$, $K_{I I I C}$ are to be determined by experiments.

The coefficients $c_{j}$ (generalized stress intensity factors, in short GSIF's) in (1) and $c_{j}(z)$ (generalized stress intensity distributions, in short GSID's) in (2) describe the intensity of stress concentrations in the neighbourhood of geometrical and structural singularities. Furthermore, the GSIF's can be used to determine the approximate shape of the plastic zone in elasto-plastic materials [13].

Due to its great importance in the applications, the computation of the GSIF's and the GSID's attracted much attention of both engineers and mathematicians. In most applications, the GSIF's and the GSID's are calculated by extraction from displacement or stress fields or by evaluating certain path integrals [13]. An alternative approach is the weight function method introduced for $2 \mathrm{D}$ crack problems in homogeneous materials by Bueckner [4] and Rice [26] and extended later to more complicated geometries. The weight functions are particular elastic fields of infinite energy and can be calculated semi-analytically for 2D $[7,14]$ and numerically for 3D problems [2]. Maz'ya and Plamenevsky [19] have derived coefficient formulas (so-called Maz'ya/Plamenevsky functionals) for general elliptic boundary value problems in domains with corners, in which general weight functions appear. This approach was generalized by Maz'ya and Rossmann [20], Rossmann and Sändig [28] for the pointwise evaluation of the GSID's $c_{i}(\cdot)$. Unfortunately, the corresponding weight functions are known analytically only for special geometries, e.g. for penny-shaped and half-plane cracks in infinite domains [5]. A numerical approach to the computation of 3D weight functions with point singularities can be found in $[2,29]$. Moreover, numerical methods for the computation of GSIF's in bonded materials were recently developed $[30,34]$.

The application of Maz'ya-Plamenevsky functionals for the numerical approximation of stress intensity factors for two-dimensional boundary value problems with the help of FEM is widely analysed in the literature (see $[1,3,8])$, where error estimates for the FEM approximation are given. 


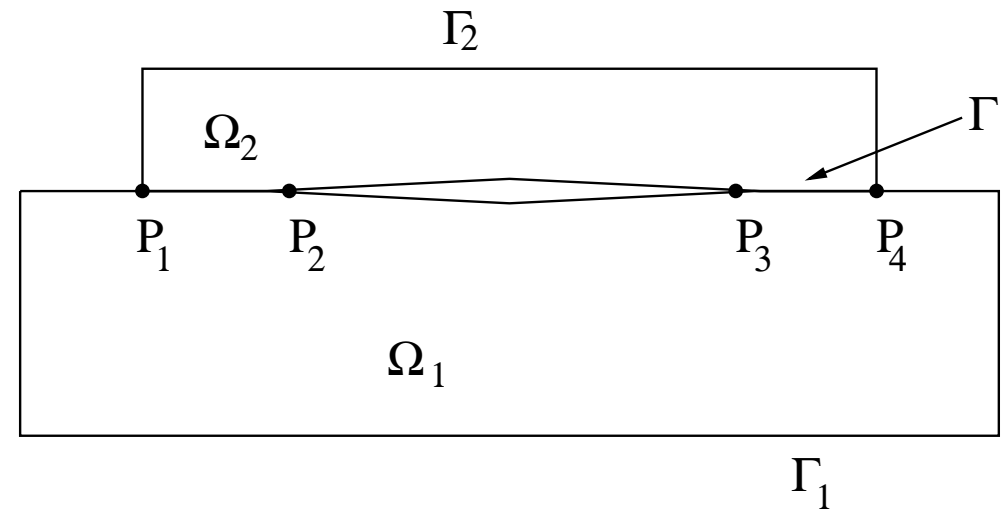

Figure 1. 2D coupled structure semiconductor.

In this paper we study 2D and 3D problems for bonded elastic structures. In the 2D case we adapt coefficient formulas and their proofs, known for homogeneous materials, to transmission problems. New is here the computation of the coefficients with boundary element methods and the corresponding error analysis based on the Aubin-Nitsche trick. Numerical examples illustrate the efficiency of the method and we compare the results with those obtained by other numerical methods. The weight functions in the adapted Maz'ya/Plamenevsky functionals are calculated semi-analytically as it was done in $[7,14]$. In the $3 \mathrm{D}$ case we present for three-dimensional plane interface crack problems a method for the computation of the integrals

$$
\int_{M} c_{i}(s) h(s) \mathrm{d} s
$$

with test functions $h(\cdot)$ defined on the crack front. In this case the weight functions have a uniform singularity along the whole edge $M$ and can be derived from the known weight functions for $2 \mathrm{D}$ problems. This idea appeared already in $[17,21]$ for harmonic problems in simple domains but was not further investigated. We underline, that in this way we can avoid the computation of the $3 \mathrm{D}$ dual singular functions with point singularities which is numerically expensive.

The paper is organized as follows: Section 2 is devoted to the formulation of the problem. In Section 3 we describe the computation of $2 \mathrm{D}$ singular solutions, which are necessary for the description of the asymptotic expansions and the construction of the weight functions. Then we give in Section 4 asymptotic expansions of elastic fields for 2D interface problems and derive several formulas for the generalized stress intensity factors using weight function techniques. Next, we apply the coefficient formulas to the approximation of the GSIF's and give corresponding error estimates along with some numerical results. Finally, we apply in Section 6 the 2D singular solutions in order to create practicable formulas for the computation of stress intensity distributions for $3 \mathrm{D}$ plane interface cracks.

\section{Formulation of the Problem}

Let $\Omega_{1}, \Omega_{2} \subset \mathbb{R}^{2}$, be two bounded domains such that $\partial \Omega_{1} \cap \partial \Omega_{2} \neq \emptyset$ (see Fig. 1). We denote by $\Gamma \subset \partial \Omega_{1} \cap \partial \Omega_{2}$ the interface and by $\Gamma_{i}=\partial \Omega_{i} \backslash \bar{\Gamma}$ the outer boundary pieces.

One of the domains $\Omega_{i}$ can be empty. If $\Gamma$ is a proper subset of $\partial \Omega_{1} \cap \partial \Omega_{2}$, then interface cracks occur. Let us denote by $P_{j}$ corner points with $P_{j} \subset \partial \Gamma$ and assume that every point $P_{j}$ has a neighbourhood $\mathcal{U}_{j}$ such that $\mathcal{U}_{j} \cap \Omega_{i}$ is diffeomorph to the intersection of the infinite wedge $C_{i}\left(P_{j}\right)$ with the unit ball $B_{1}\left(P_{j}\right)$ centred in $P_{j}, i=1,2$. 
The infinite wedge $C_{i}\left(P_{j}\right)$ is defined by

$$
C_{i}\left(P_{j}\right)=\left\{(r, \omega) \in \mathbb{R}^{2}: r=\left|x-P_{j}\right|, \omega_{i-1}<\omega<\omega_{i}\right\}, i=1,2 .
$$

The domains $\Omega_{i}$ are occupied by isotropic linear elastic materials. The linearized elasticity equations for the displacement vectors $u_{i}=\left(u_{i}^{1}, u_{i}^{2}\right)$ in $\Omega_{i}$ read

$$
L_{i} u_{i}(x):=\mu_{i} \Delta u_{i}(x)+\left(\lambda_{i}+\mu_{i}\right) \nabla \operatorname{div} u_{i}(x)=f_{i}(x) .
$$

On the boundary pieces $\Gamma_{i}^{D}, i=1,2$, Dirichlet conditions are given

$$
u_{i}=g_{i}, i=1,2 \text {, }
$$

whereas on $\Gamma_{i}^{N}$ boundary stresses are prescribed

$$
\sigma_{i}\left(u_{i}\right) \vec{n}_{i}=h_{i}, i=1,2
$$

where the components of the stress tensor $\sigma_{i}\left(u_{i}\right)$ are given by

$$
\sigma_{i}^{k, l}\left(u_{i}\right)=\lambda_{i}\left(\sum_{j=1}^{2} \partial_{j} u_{i}^{j}\right) \delta_{k l}+\mu_{i}\left(\partial_{k} u_{i}^{l}+\partial_{l} u_{i}^{k}\right)
$$

where $\vec{n}_{i}$ is the exterior normal vector on $\partial \Omega_{i}$. Assuming that standard transmission conditions are satisfied on the interface $\Gamma$ we obtain the boundary transmission problem

$$
\begin{aligned}
\mu_{i} \Delta u_{i}+\left(\lambda_{i}+\mu_{i}\right) \nabla \operatorname{div} u_{i} & =f_{i} & & \text { in } \Omega_{i}, \\
u_{i} & =g_{i} & & \text { on } \Gamma_{i}^{D}, \\
\sigma_{i}\left(u_{i}\right) \vec{n}_{i} & =h_{i} & & \text { on } \Gamma_{i}^{N}, \\
u_{1}-u_{2} & =p_{1} & & \text { on } \Gamma, \\
\sigma_{1}\left(u_{1}\right) \vec{n}_{1}+\sigma_{2}\left(u_{2}\right) \vec{n}_{2} & =p_{2} & & \text { on } \Gamma .
\end{aligned}
$$

The behaviour of the two-dimensional displacement fields $u_{1}, u_{2}$ in a neighbourhood of a corner point $P$ can be described by an asymptotic expansion, provided the right-hand sides of (7) are sufficiently smooth and satisfy some compatibility conditions in $P[25]$ :

$$
\left(\begin{array}{l}
u_{1} \\
u_{2}
\end{array}\right)(r, \omega)=\sum_{j=1}^{N} \sum_{k=1}^{m_{g}\left(\alpha_{j}\right)} \sum_{l=0}^{\kappa_{k, j}-1} c_{j, k, l}\left(\begin{array}{c}
v_{1, j}^{l, k} \\
v_{2, j}^{l, k}
\end{array}\right)(r, \omega)+\left(\begin{array}{c}
v_{1} \\
v_{2}
\end{array}\right)(r, \omega),
$$

where

$$
\left(\begin{array}{c}
v_{1, j}^{l, k} \\
v_{2, j}^{l, k}
\end{array}\right)(r, \omega)=r^{\alpha_{j}} \sum_{s=0}^{l} \frac{1}{s !}(\log r)^{s}\left(\begin{array}{c}
\varphi_{1, j}^{l-s, k} \\
\varphi_{2, j}^{l-s, k}
\end{array}\right)(\omega)
$$

and $r=|x-P|$. The complex exponents $\alpha_{j}$ and the functions $\left(\varphi_{1, j}^{l, k}, \varphi_{2, j}^{l, k}\right)$ are eigenvalues and eigensolutions (elements of Jordan-chains) of a corresponding generalized eigenvalue problem (see Sect. 3). $N$ is the number of eigenvalues in the strip $0<\operatorname{Re} \alpha<d, m_{g}\left(\alpha_{j}\right)$ denotes the number of Jordan-chains to the eigenvalue $\alpha_{j}$ (geometrical multiplicity) and $\kappa_{k, j}$ is the length of the $k$ th Jordan-chain. In the following we will assume for simplicity that the Jordan-chains have the length 1, i.e. no logarithmic terms appear in (8) and we restrict our considerations to $d=1$. 




FIGURE 2. Interface crack.

Furthermore we consider three-dimensional coupled elastic structures with a smooth plane interface crack (see Fig. 2). The asymptotics of the displacement fields $u_{1}, u_{2}$ in the neighbourhood of the crack front $M$ has the form

$$
\begin{aligned}
\left(\begin{array}{l}
u_{1} \\
u_{2}
\end{array}\right)(r, \omega, s)= & c_{1}(s) r^{1 / 2}\left(\begin{array}{l}
\varphi_{1,1}(\omega) \\
\varphi_{2,1}(\omega)
\end{array}\right)+c_{2}(s) r^{1 / 2+i \varepsilon}\left(\begin{array}{l}
\varphi_{1,2}(\omega) \\
\varphi_{2,2}(\omega)
\end{array}\right) \\
& +c_{3}(s) r^{1 / 2-i \varepsilon}\left(\begin{array}{l}
\varphi_{1,3}(\omega) \\
\varphi_{2,3}(\omega)
\end{array}\right)+\eta\left(\begin{array}{l}
w_{1} \\
w_{2}
\end{array}\right)(r, \omega, s),
\end{aligned}
$$

provided the right-hand sides of (7) are sufficiently smooth and some compatibility conditions are satisfied (see Sect. 4 for details).

Here, $s$ is the arclength on the edge $M \subset \partial \Gamma$ and $(r, \omega)$ are the local polar coordinates in the plane orthogonal to $M$ in the point $P(s)$ with parameter $s$ and the constants $\varepsilon, \beta, \nu_{i}$ are given by [36]

$$
\varepsilon=\frac{1}{2 \pi} \ln \frac{1+\beta}{1-\beta}, \quad \beta=\frac{\mu_{2}\left(1-2 \nu_{1}\right)-\mu_{1}\left(1-2 \nu_{2}\right)}{2\left[\mu_{2}\left(1-\nu_{1}\right)+\mu_{1}\left(1-\nu_{2}\right)\right]}, \quad \nu_{i}=\frac{\lambda_{i}}{2\left(\lambda_{i}+\mu_{i}\right)} .
$$

Our goal is to derive formulas for the computation of the coefficients $c_{j, k, l}$ in (8) and $c_{j}(s)$ in (10) and to use them for the numerical approximation of the coefficients.

\section{OpERATOR PENCILS AND SINGULAR FUNCTIONS}

As mentioned before, the singular functions appearing in the asymptotics (8) and (10) are generated by the Jordan-chains of some operator pencils which will be derived now. We refer the reader to [23] for main definitions and facts from the theory of operator pencils.

We create for the two-dimensional boundary transmission problem (7) an operator pencil corresponding to the corner point $P \in \bar{\Gamma} \cap \bar{\Gamma}_{1} \cap \bar{\Gamma}_{2}$. To this end we map a neighbourhood $\mathcal{V}(P):=\mathcal{U}(P) \cap\left(\bar{\Omega}_{1} \cup \bar{\Omega}_{2}\right)$ of the point $P$ by a diffeomorphism $\chi$ onto $\chi(\mathcal{V}(P))=\left[\overline{C_{1}(P)} \cup \overline{C_{2}(P)}\right] \cap B_{1}(P)$ (see Fig. 3).

Let $\chi\left(x_{1}, x_{2}\right)=\left(y_{1}, y_{2}\right)$ be the new coordinates in $\left[\overline{C_{1}(P)} \cup \overline{C_{2}(P)}\right] \cap B_{1}(P)$. Writing the boundary transmission problem $(7)$ in the curvilinear coordinates $\left(y_{1}, y_{2}\right)$ we obtain a boundary transmission problem for differential 


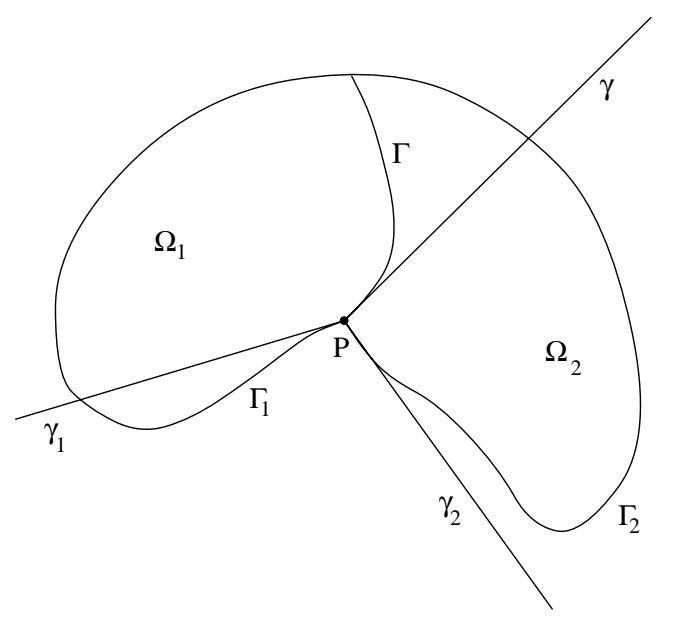

FiguRE 3. Neighbourhood of the corner point P.

operators with variable coefficients, which we denote shortly by

$$
\mathcal{A}\left(y, D_{y}\right)=\left\{\mathcal{L}_{i}\left(y, D_{y}\right), \mathcal{B}_{i}\left(y, D_{y}\right), \mathcal{T}\left(y, D_{y}\right), i=1,2\right\} .
$$

It is well known from the theory of elliptic boundary value problems with variable coefficients [15] that the behaviour of the solutions to elliptic boundary value problems in the neighbourhood of the corner point $P$ is essentially influenced by the behaviour of solutions to an elliptic boundary value problem generated by the principal part of the operator $\mathcal{A}\left(y, D_{y}\right)$ with coefficients frozen in the point $P$. Moreover, the leading terms in the asymptotics (8) have the same structure as in the case of operators with constant coefficients. We extend these ideas to boundary transmission problems (see [24]). Since the principal part of the operator $\mathcal{A}$ with coefficients frozen in the corner point $P$ coincides with the original Lamé and boundary operators, we study the boundary transmission problem in the double wedge $\overline{C_{1}(P)} \cup \overline{C_{2}(P)}$ with vanishing right-hand sides

$$
\begin{aligned}
& L_{i} u_{i}=0 \quad \text { in } C_{i}(P), \\
& u_{1}-u_{2}=0 \quad \text { on } \gamma \text {, } \\
& \sigma_{1}\left(u_{1}\right) \vec{n}_{1}+\sigma_{2}\left(u_{2}\right) \vec{n}_{2}=0 \quad \text { on } \gamma \\
& B_{i} u_{i}:=u_{i} \quad=0 \quad \text { on } \gamma_{i}, \quad(D-D) \\
& \text { or } B_{i} u_{i}:=\sigma_{i}\left(u_{i}\right) \vec{n}_{i}=0 \quad \text { on } \gamma_{i}, \quad(N-N)
\end{aligned}
$$

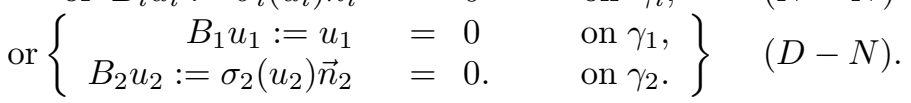

Writing the boundary transmission problem in polar coordinates and applying the Mellin transform

$$
\mathcal{M} u(\alpha, \omega)=\int_{0}^{\infty} r^{-\alpha-1} u(r, \omega) \mathrm{d} r
$$


with respect to the distance $r=|x-P|$ we get a boundary transmission problem for a system of ordinary differential equations depending on a complex parameter $\alpha$

$$
\begin{aligned}
\mathbb{L}_{i}(\alpha) u_{i}(\alpha, \omega) & =0 \quad \text { for } \omega_{i-1}<\omega<\omega_{i}, i=1,2, \\
\mathbb{B}_{1}(\alpha) u_{1}\left(\alpha, \omega_{0}\right) & =0 \\
\mathbb{B}_{2}(\alpha) u_{2}\left(\alpha, \omega_{2}\right) & =0 \\
u_{1}\left(\alpha, \omega_{1}\right)-u_{2}\left(\alpha, \omega_{1}\right) & =0 \\
\mathbf{T}_{1}(\alpha) u_{1}\left(\alpha, \omega_{1}\right)-\mathbf{T}_{2}(\alpha) u_{2}\left(\alpha, \omega_{1}\right) & =0 .
\end{aligned}
$$

Here (see e.g. [14])

$$
\begin{aligned}
\mathbf{L}_{i}(\alpha)\left(\begin{array}{c}
u_{i}^{r} \\
u_{i}^{\omega}
\end{array}\right)=\left(\begin{array}{c}
\tau_{i} \partial_{\omega}^{2} u_{i}^{r}+\left(\alpha^{2}-1\right) u_{i}^{r}+\left[(\alpha-1)-\tau_{i}(\alpha+1)\right] \partial_{\omega} u_{i}^{\omega} \\
\partial_{\omega}^{2} u_{i}^{\omega}+\tau_{i}\left(\alpha^{2}-1\right) u_{i}^{\omega}+\left[(\alpha+1)-\tau_{i}(\alpha-1)\right] \partial_{\omega} u_{i}^{r}
\end{array}\right), \\
\mathbf{T}_{i}(\alpha)\left(\begin{array}{c}
u_{i}^{r} \\
u_{i}^{\omega}
\end{array}\right)=\left(\begin{array}{c}
\mu_{i}\left(\partial_{\omega} u_{i}^{r}+(\alpha-1) u_{i}^{\omega}\right) \\
\left(\lambda_{i}+2 \mu_{i}\right) \partial_{\omega} u_{i}^{\omega}+\left(\lambda_{i}(\alpha+1)+2 \mu_{i}\right) u_{i}^{r}
\end{array}\right), \\
\mathbb{B}_{i}(\alpha)\left(\begin{array}{c}
u_{i}^{r} \\
u_{i}^{\omega}
\end{array}\right)=\left\{\begin{array}{c}
\left(\begin{array}{c}
u_{i}^{r} \\
u_{i}^{\omega}
\end{array}\right) \text { if } \quad B_{i} u_{i}=u_{i}, \\
\mathbf{T}_{i}(\alpha)\left(\begin{array}{c}
u_{i}^{r} \\
u_{i}^{\omega}
\end{array}\right) \text { if } \quad B_{i} u_{i}=\sigma_{i}\left(u_{i}\right) \vec{n}
\end{array}\right. \\
\tau_{i}=\mu_{i} /\left(\lambda_{i}+2 \mu_{i}\right)
\end{aligned}
$$

and $\left(u_{i}^{r}, u_{i}^{\omega}\right)$ are the polar components of the displacement vector $u_{i}$.

For every $\alpha \in \mathbb{C}$ the operator $\mathcal{A}_{L}(\alpha)$ defined by

$$
\begin{array}{r}
\mathcal{A}_{L}(\alpha)\left(\begin{array}{l}
u_{1} \\
u_{2}
\end{array}\right)=\left\{\left(\begin{array}{cc}
\mathbf{L}_{1}(\alpha) u_{1} & 0 \\
0 & \mathbf{L}_{2}(\alpha) u_{2}
\end{array}\right)\right. \\
\left(\begin{array}{cc}
\mathbb{B}_{1}(\alpha) u_{1}\left(\omega_{0}\right) & 0 \\
0 & \mathbb{B}_{2}(\alpha) u_{2}\left(\omega_{2}\right)
\end{array}\right), \\
\left.\left(\begin{array}{cc}
u_{1}\left(\omega_{1}\right) & -u_{2}\left(\omega_{1}\right) \\
\mathbf{T}_{1}(\alpha) u_{1}\left(\omega_{1}\right) & -\mathbf{T}_{2}(\alpha) u_{2}\left(\omega_{1}\right)
\end{array}\right)\right\}
\end{array}
$$

maps $\left[H^{2}\left(\omega_{0}, \omega_{1}\right)\right]^{2} \times\left[H^{2}\left(\omega_{1}, \omega_{2}\right)\right]^{2}$ into $\left[L_{2}\left(\omega_{0}, \omega_{1}\right)\right]^{2} \times\left[L_{2}\left(\omega_{1}, \omega_{2}\right)\right]^{2} \times\left[\mathbb{C}^{2} \times \mathbb{C}^{2}\right] \times\left[\mathbb{C}^{2} \times \mathbb{C}^{2}\right]$.

The eigenvalues and the eigenfunctions of the operator pencil $\mathcal{A}_{L}(\cdot)$ can be easily calculated using the idea from $[7,14]$. It is known [14] that the solutions of the homogeneous equations

$$
\mathbb{L}_{i}(\alpha) u_{i}(\omega)=0
$$

have the form

$$
\begin{aligned}
\left(\begin{array}{c}
u_{i}^{r} \\
u_{i}^{\omega}
\end{array}\right)(r, \omega)= & r^{\alpha}\left[A_{i}\left(\begin{array}{c}
\cos ((1+\alpha) \omega) \\
-\sin ((1+\alpha) \omega)
\end{array}\right)+B_{i}\left(\begin{array}{c}
\sin ((1+\alpha) \omega) \\
\cos ((1+\alpha) \omega)
\end{array}\right)\right. \\
& \left.+C_{i}\left(\begin{array}{c}
\cos ((1-\alpha) \omega) \\
-\zeta_{i} \sin ((1-\alpha) \omega)
\end{array}\right)+D_{i}\left(\begin{array}{c}
\sin ((1-\alpha) \omega) \\
\zeta_{i} \cos ((1-\alpha) \omega)
\end{array}\right)\right] \\
=: & r^{\alpha}\left[A_{i} U_{1}(\alpha, \omega)+B_{i} U_{2}(\alpha, \omega)+C_{i} U_{3}(\alpha, \omega)+D_{i} U_{4}(\alpha, \omega)\right]
\end{aligned}
$$

where $\zeta_{i}=\left(\kappa_{i}+\alpha\right) /\left(\kappa_{i}-\alpha\right)$ and $\kappa_{i}=\left(3 \mu_{i}+\lambda_{i}\right) /\left(\mu_{i}+\lambda_{i}\right)$. We insert the ansatz (15) into (13) and obtain a system of 8 homogeneous linear equations for the unknown coefficients $A_{i}, B_{i}, C_{i}, D_{i}, i=1,2$. The corresponding 
parameter depending matrix $A_{L}(\alpha)$ has the form

$$
A_{L}(\alpha)=\left(\begin{array}{cc}
U_{1}(\alpha, 0) & 0 \\
U_{1}\left(\alpha, \omega_{1}\right) & -U_{2}\left(\alpha, \omega_{1}\right) \\
\mathbf{T}_{1}(\alpha) U_{1}\left(\alpha, \omega_{1}\right) & -\mathbf{T}_{2}(\alpha) U_{2}\left(\alpha, \omega_{1}\right) \\
0 & U_{2}\left(\alpha, \omega_{2}\right)
\end{array}\right) .
$$

Thus the problem (13) has a non-trivial solution iff the matrix $A_{L}(\alpha)$ is singular, i.e. the spectrum of the operator pencil $\mathcal{A}_{L}$ consists of all complex-valued roots of the transcendent equation

$$
\operatorname{det} A_{L}(\alpha)=0
$$

Suppose that $\alpha_{j}$ is a root of (17) and that the coefficient vector

$$
v:=\left(A_{1}, B_{1}, C_{1}, D_{1}, A_{2}, B_{2}, C_{2}, D_{2}\right)
$$

satisfies the equation

$$
A_{L}\left(\alpha_{j}\right) v=0
$$

Then

$$
\varphi_{i}:=r^{\alpha_{j}}\left[A_{i} U_{1}(\alpha, \omega)+B_{i} U_{2}(\alpha, \omega)+C_{i} U_{3}(\alpha, \omega)+D_{i} U_{4}(\alpha, \omega)\right], i=1,2,
$$

is an eigenfunction of the operator pencil $\mathcal{A}_{L}$ to the eigenvalue $\alpha_{j}$. Since eigenfunctions are determined up to a constant factor we will assume in the following that the coefficient vector $v$ has the Euclidean norm equal to 1 .

Harmonic fields governed by boundary value problems for the Poisson equation can be handled analogously. The problem (12) reads then

$$
\begin{array}{rllll}
\mu_{i} \Delta u_{i} & = & 0 & \text { in } C_{i}(P), & \\
u_{1}-u_{2} & = & 0 & \text { on } \gamma, \\
\mu_{1} \frac{\partial u_{1}}{\partial \vec{n}_{1}}+\mu_{2} \frac{\partial u_{2}}{\partial \vec{n}_{2}} & = & 0 & \text { on } \gamma, & \\
u_{i} & = & 0 & \text { on } \gamma_{i}, & (D-D) \\
\text { or } \mu_{i} \frac{\partial u_{i}}{\partial \vec{n}_{i}} & = & 0 & \text { on } \gamma_{i}, & (N-N) \\
\text { or }\left\{\begin{array}{rlll}
u_{1} & & 0 & \text { on } \gamma_{1}, \\
\mu_{2} \frac{\partial u_{2}}{\partial \vec{n}_{2}} & = & 0 & \text { on } \gamma_{2} .
\end{array}\right\} & (D-N) .
\end{array}
$$

Applying again the Mellin transform we obtain

$$
\begin{aligned}
l_{i}(\alpha) u_{i}(\alpha, \omega):=\frac{\partial^{2} u_{i}}{\partial \omega^{2}}(\alpha, \omega)+\alpha^{2} u_{i}(\omega) & =0 \quad \text { for } \quad \omega_{i-1}<\omega<\omega_{i}, i=1,2, \\
u_{1}\left(\alpha, \omega_{1}\right)-u_{2}\left(\alpha, \omega_{1}\right) & =0 \\
\mu_{1} \frac{\partial u_{1}}{\partial \omega}\left(\alpha, \omega_{1}\right)-\mu_{2} \frac{\partial u_{2}}{\partial \omega}\left(\alpha, \omega_{1}\right) & =0 \\
b_{1}(\alpha) u_{1}\left(\alpha, \omega_{0}\right) & =0 \\
b_{2}(\alpha) u_{2}\left(\alpha, \omega_{2}\right) & =0 .
\end{aligned}
$$


Here is

$$
b_{i}(\alpha) u_{i}\left(\alpha, \omega_{j}\right)= \begin{cases}u_{i}\left(\omega_{j}\right) & \text { for Dirichlet boundary conditions } \\ \mu_{i} \frac{\partial}{\partial \omega} u_{i}\left(\alpha, \omega_{j}\right) & \text { for Neumann boundary conditions. }\end{cases}
$$

The corresponding operators $\mathcal{A}_{\Delta}(\alpha)$ defined by

$$
\mathcal{A}_{\Delta}(\alpha)\left(\begin{array}{l}
u_{1} \\
u_{2}
\end{array}\right)=\left\{\left(\begin{array}{cc}
l_{1}(\alpha) u_{1} & 0 \\
0 & l_{2}(\alpha) u_{2}
\end{array}\right),\left(\begin{array}{cc}
b_{1}(\alpha) u_{1}\left(\omega_{0}\right) & 0 \\
0 & b_{2}(\alpha) u_{2}\left(\omega_{2}\right)
\end{array}\right),\left(\begin{array}{cc}
u_{1}\left(\omega_{1}\right) & -u_{2}\left(\omega_{1}\right) \\
\mu_{1} \frac{\partial}{\partial \omega} u_{1}\left(\omega_{1}\right) & -\mu_{2} \frac{\partial}{\partial \omega} u_{2}\left(\omega_{1}\right)
\end{array}\right)\right\}
$$

$\operatorname{map} H^{2}\left(\omega_{0}, \omega_{1}\right) \times H^{2}\left(\omega_{1}, \omega_{2}\right)$ into $L_{2}\left(\omega_{0}, \omega_{1}\right) \times L_{2}\left(\omega_{1}, \omega_{2}\right) \times[\mathbb{C} \times \mathbb{C}] \times[\mathbb{C} \times \mathbb{C}]$ for every $\alpha \in \mathbb{C}$. The eigenvalues and eigenfunctions of the operator pencil $\mathcal{A}_{\Delta}$ can be calculated as before taking into account that the eigenfunctions have the general form

$$
u_{i}(\alpha, \omega)=r^{\alpha}\left[A_{i} \sin (\alpha \omega)+B_{i} \cos (\alpha \omega)\right]
$$

with constants $A_{i}, B_{i}, i=1,2$, to be determined.

\section{Asymptotic expansions And COEFFicient Formulas. 2D PROBlems}

\subsection{Asymptotic expansions}

The eigenvalues and corresponding Jordan-chains of eigensolutions and associate eigensolutions of the operator pencils $(14,22)$ govern the asymptotic expansions $(8,10)$. Here we cite the results for two-dimensional corner singularities.

Let us assume for the sake of simplicity that all Jordan-chains of the operator pencil $\mathcal{A}_{L}$ have the length 1 . This assumption is valid apart from some isolated opening angles. We denote by $\left(\varphi_{1, j}, \varphi_{2, j}\right)$ an eigenfunction corresponding to the eigenvalue $\alpha_{j}$ and define

$$
\left(\begin{array}{c}
v_{1, j} \\
v_{2, j}
\end{array}\right)(r, \omega)=r^{\alpha_{j}}\left(\begin{array}{c}
\varphi_{1, j} \\
\varphi_{2, j}
\end{array}\right)(\omega)
$$

In what follows we use trace spaces defined on an open part of the boundary

$$
\tilde{H}^{s}\left(\Gamma_{i}\right):= \begin{cases}\left\{u \in H^{s}\left(\partial \Omega_{i}\right): \operatorname{supp} u \subset \bar{\Gamma}_{i}\right\} & \text { for } \quad s \geq 0 \\ \left(H^{-s}\left(\Gamma_{i}\right)\right)^{\prime} & \text { for } \quad s<0\end{cases}
$$

Theorem 4.1 ([24]). Let $f_{i} \in\left[L_{2}\left(\Omega_{i}\right)\right]^{2}, \quad g_{i} \in\left[H^{3 / 2}\left(\Gamma_{i}^{D}\right)\right]^{2}, \quad h_{i} \in\left[\tilde{H}^{1 / 2}\left(\Gamma_{i}^{N}\right)\right]^{2}, \quad p_{1} \in\left[H^{3 / 2}(\Gamma)\right]^{2}, \quad p_{2} \in$ $\left[\tilde{H}^{1 / 2}(\Gamma)\right]^{2}$. Furthermore we assume that there exist functions $w_{i} \in\left[H^{2}\left(\Omega_{i}\right)\right]^{2}$ with

$$
\begin{aligned}
\left.w_{i}\right|_{\Gamma_{i}^{D}} & =\left.g_{i}\right|_{\Gamma_{i}^{D}}, \\
\left.\sigma_{i}\left(w_{i}\right) \vec{n}_{i}\right|_{\Gamma_{i}^{N}} & =\left.h_{i}\right|_{\Gamma_{i}^{N}}, \\
\left.\left(w_{1}-w_{2}\right)\right|_{\Gamma} & =\left.p_{1}\right|_{\Gamma}, \\
\left.\left(\sigma_{1}\left(w_{1}\right) \vec{n}_{1}+\sigma_{2}\left(w_{2}\right) \vec{n}_{2}\right)\right|_{\Gamma} & =\left.p_{2}\right|_{\Gamma} .
\end{aligned}
$$

Then the solution of the two-dimensional problem (7) admits the decomposition

$$
\left(\begin{array}{l}
u_{1} \\
u_{2}
\end{array}\right)(r, \omega)=\sum_{0<\operatorname{Re}\left(\alpha_{j}\right)<1} c_{j} r^{\alpha_{j}}\left(\begin{array}{c}
\varphi_{1, j} \\
\varphi_{2, j}
\end{array}\right)(r, \omega)+\left(\begin{array}{l}
v_{1} \\
v_{2}
\end{array}\right)(r, \omega)
$$


with $v_{i} \in\left[H^{2}\left(\Omega_{i}\right)\right]^{2}$. The summation in (25) is taken over all eigenvalues $\alpha_{j}$ corresponding to their geometric multiplicity $m_{g}\left(\alpha_{j}\right)$.

Remark 4.1. The existence of functions $w_{i}$ in Theorem 4.1 induces compatibility conditions between the data $g_{i}, h_{i}$ and $p_{i}$ in the point $P$. For example, in case of the D-D problem we have for sufficiently smooth data

$$
\lim _{x \in \Gamma_{1}, x \rightarrow P} g_{1}(x)-\lim _{x \in \Gamma_{2}, x \rightarrow P} g_{2}(x)=\lim _{x \in \Gamma, x \rightarrow P} p_{1}(x) .
$$

\subsection{Coefficient formulas for stress intensity factors}

First we derive formulas for the coefficients $c_{j}$ in (25). For the sake of simplicity we assume in the proofs that the boundary pieces $\Gamma_{i}$ and the interface $\Gamma$ consist of straight lines in a neighbourhood of the corner point $P$.

Let $\alpha_{j}$ be an eigenvalue of the operator pencil $\mathcal{A}_{L}$ with the eigenfunction $\left(\varphi_{1, j}, \varphi_{2, j}\right)$. It can be easily shown that $-\bar{\alpha}$ is an eigenvalue of $\mathcal{A}_{L}$ iff $\alpha$ is also an eigenvalue of $\mathcal{A}_{L}$. We introduce the notation

$$
\left(\begin{array}{l}
w_{1, j} \\
w_{2, j}
\end{array}\right)(r, \omega)=r^{-\overline{\alpha_{j}}}\left(\begin{array}{c}
\psi_{1, j} \\
\psi_{2, j}
\end{array}\right)(\omega)
$$

where $\left(\psi_{1, j}, \psi_{2, j}\right)$ is an eigenfunction of the operator pencil $\mathcal{A}_{L}$ corresponding to the eigenvalue $-\bar{\alpha}_{j}$. Thus the functions $\left(w_{1, j}, w_{2, j}\right)$ are singular solutions (weight functions) of the homogeneous problem (12).

Further, we define for complex valued functions $u_{i}, v_{i}: \Omega_{i} \rightarrow \mathbb{C}$, which are smooth enough, the sesquilinear functional $q_{i}$ by

$$
q_{i}\left(u_{i}, v_{i}\right)=\int_{\Omega_{i}} L_{i} u_{i} \cdot \overline{v_{i}} \mathrm{~d} x-\int_{\Omega_{i}} u_{i} \cdot \overline{L_{i} v_{i}} \mathrm{~d} x+\int_{\partial \Omega_{i}} u_{i} \cdot \overline{\sigma_{i}\left(v_{i}\right) \vec{n}_{i}} \mathrm{~d} s_{x}-\int_{\partial \Omega_{i}} \sigma_{i}\left(u_{i}\right) \vec{n}_{i} \cdot \overline{v_{i}} \mathrm{~d} s_{x} .
$$

Note that $q_{i}\left(u_{i}, v_{i}\right)=0$ for sufficiently smooth functions $u_{i}, v_{i}$ due to Green's formula.

In the following we denote by $\eta \in C_{0}^{\infty}\left(\mathbb{R}_{0}^{+}\right)$a cut-off function with $\eta(r)=1$ for $0 \leq r \leq r_{0}$.

Lemma 4.2. Let $\left(v_{1, j}, v_{2, j}\right)$ and $\left(w_{1, j}, w_{2, j}\right)$ be the functions defined by (24) and (26), respectively. Then

$$
q_{i}\left(\eta v_{i, j}, w_{i, j}\right)=\int_{\partial B_{\delta}(P) \cap \Omega_{i}}\left(\sigma_{i}\left(v_{i, j}\right) \vec{n}_{i} \cdot \overline{w_{i, j}}-v_{i, j} \cdot \overline{\sigma_{i}\left(w_{i, j}\right) \vec{n}_{i}}\right) \mathrm{d} s_{x}
$$

with small enough $\delta>0$.

Proof. Since $v_{i, j}, w_{i, j} \in\left[H^{2}\left(\Omega_{i} \backslash B_{\delta}(P)\right)\right]^{2}$ we can apply Green's formula in $\Omega_{i} \backslash B_{\delta}(P)$ and take the limit as $\delta \rightarrow 0$. In this way we obtain

$$
q_{i}\left(\eta v_{i, j}, w_{i, j}\right)=\lim _{\delta \rightarrow 0} \int_{\partial B_{\delta}(P) \cap \Omega_{i}}\left(\sigma_{i}\left(v_{i, j}\right) \vec{n}_{i} \cdot \overline{w_{i, j}}-v_{i, j} \cdot \overline{\sigma_{i}\left(w_{i, j}\right) \vec{n}_{i}}\right) \mathrm{d} s_{x}
$$

Let us prove that the value of the integral on the right-hand side of (29) does not depend on the choice of $\delta>0$. Let $0<\delta_{1}<\delta_{2}$ and $B_{\delta_{1}, \delta_{2}}=\left[B_{\delta_{2}}(P) \backslash B_{\delta_{1}}(P)\right] \cap \Omega_{i}$. Since the functions $v_{i, j}, w_{i, j}$ are smooth in $\overline{B_{\delta_{1}, \delta_{2}}}$ we obtain due to Green's formula

$$
\int_{\partial B_{\delta_{1}, \delta_{2}}}\left(\sigma_{i}\left(v_{i, j}\right) \vec{n}_{i} \cdot \overline{w_{i, j}}-v_{i, j} \cdot \overline{\sigma_{i}\left(w_{i, j}\right) \vec{n}_{i}}\right) \mathrm{d} s_{x}=0
$$


The functions $v_{i, j}, w_{i, j}$ satisfy homogeneous boundary conditions on $\partial B_{\delta_{1}, \delta_{2}} \cap \partial \Omega_{i}$. Therefore we conclude that

$$
\int_{\partial B_{\delta_{1}}(P) \cap \Omega_{i}}\left(\sigma_{i}\left(v_{i, j}\right) \vec{n}_{i} \cdot \overline{w_{i, j}}-v_{i, j} \cdot \overline{\sigma_{i}\left(w_{i, j}\right) \vec{n}_{i}}\right) \mathrm{d} s_{x}=\int_{\partial B_{\delta_{2}}(P) \cap \Omega_{i}}\left(\sigma_{i}\left(v_{i, j}\right) \vec{n}_{i} \cdot \overline{w_{i, j}}-v_{i, j} \cdot \overline{\sigma_{i}\left(w_{i, j}\right) \vec{n}_{i}}\right) \mathrm{d} s_{x} .
$$

Since the eigenfunctions $\left(\psi_{1, j}, \psi_{2, j}\right)$ are defined up to a constant factor we impose the following normalization condition:

Lemma 4.3. Let the eigenfunctions $\left\{\left(\varphi_{1, j}, \varphi_{2, j}\right), 1 \leq j \leq m_{g}(\alpha)\right\}$ to the eigenvalue $\alpha$ be fixed. Then the eigenfunctions $\left\{\left(\psi_{1, k}, \psi_{2, k}\right), 1 \leq k \leq m_{g}(\alpha)\right\}$ to the eigenvalue $-\bar{\alpha}$ can be chosen in such a way that the following biorthonormality condition is satisfied

$$
\sum_{i=1}^{2} q_{i}\left(\eta v_{i, j}, w_{i, k}\right)= \begin{cases}1 & \text { if } j=k \\ 0 & \text { if } j \neq k\end{cases}
$$

Now we are in position to derive the coefficient formulas.

Theorem 4.4. Suppose that the assumptions of Theorem 4.1 are satisfied and that the functions $\left(v_{1, j}, v_{2, j}\right)$ and $\left(w_{1, j}, w_{2, j}\right)$ defined by (24) and (26), respectively, satisfy the biorthonormality condition (30). Then the coefficients $c_{j}$ in (25) are given by

$$
\begin{aligned}
c_{j}= & \sum_{i=1}^{2}\left(\int_{\Omega_{i}} L_{i}\left(\eta u_{i}\right) \cdot \overline{w_{i, j}} \mathrm{~d} x+\int_{\Gamma_{i}}\left(\eta u_{i} \cdot \overline{\sigma_{i}\left(w_{i, j}\right) \vec{n}_{i}}-\sigma_{i}\left(\eta u_{i}\right) \vec{n}_{i} \cdot \overline{w_{i, j}}\right) \mathrm{d} s_{x}\right) \\
& +\int_{\Gamma}\left(\eta p_{1} \cdot \overline{\sigma_{1}\left(w_{1, j}\right) \vec{n}_{1}}-\left(\sigma_{1}\left(\eta u_{1}\right) \vec{n}_{1}+\sigma_{2}\left(\eta u_{2}\right) \vec{n}_{2}\right) \cdot \overline{w_{1, j}}\right) \mathrm{d} s_{x} .
\end{aligned}
$$

Proof. We insert the decomposition (25) into $\sum_{i=1}^{2} q_{i}\left(\eta u_{i}, w_{i, j}\right)$ and obtain

$$
\begin{aligned}
\sum_{i=1}^{2} q_{i}\left(\eta u_{i}, w_{i, j}\right) & =\sum_{i=1}^{2} q_{i}\left(\eta \sum_{0<\operatorname{Re} \alpha_{k}<1} c_{k} v_{i, k}+\eta v_{i}, w_{i, j}\right) \\
& =\sum_{0<\operatorname{Re} \alpha_{k}<1} c_{k} \sum_{i=1}^{2} q_{i}\left(\eta v_{i, k}, w_{i, j}\right)+\sum_{i=1}^{2} q_{i}\left(\eta v_{i}, w_{i, j}\right) .
\end{aligned}
$$

Due to Green's formula the terms $q_{i}\left(\eta v_{i}, w_{i, j}\right)$ vanish. If $\alpha_{j}-\alpha_{k}>0$ then $q_{i}\left(\eta v_{i, k}, w_{i, j}\right)$ vanishes due to (29). If $\alpha_{j}-\alpha_{k}<0$ then the terms $q_{i}\left(\eta v_{i, k}, w_{i, j}\right)$ must vanish since $\sum_{i=1}^{2} q_{i}\left(\eta u_{i}, w_{i, j}\right)$ is finite. Because of the biorthonormality condition (30) it follows then

$$
\sum_{i=1}^{2} q_{i}\left(\eta u_{i}, w_{i, j}\right)=\sum_{0<\operatorname{Re} \alpha_{j}<1} c_{j} \sum_{i=1}^{2} q_{i}\left(\eta v_{i, j}, w_{i, j}\right)=c_{j}
$$


On the other hand

$$
\begin{aligned}
\sum_{i=1}^{2} q_{i}\left(\eta u_{i}, w_{i, j}\right)=\sum_{i=1}^{2} & \left(\int_{\Omega_{i}} L_{i}\left(\eta u_{i}\right) \cdot \overline{w_{i, j}} \mathrm{~d} x-\int_{\Omega_{i}} \eta u_{i} \cdot \overline{L_{i}\left(w_{i, j}\right)} \mathrm{d} x\right. \\
& \left.\quad+\int_{\Gamma_{i}} \eta u_{i} \cdot \overline{\sigma_{i}\left(w_{i, j}\right) \vec{n}_{i}} \mathrm{~d} s_{x}-\int_{\Gamma_{i}} \sigma_{i}\left(\eta u_{i}\right) \vec{n}_{i} \cdot \overline{w_{i, j}} \mathrm{~d} s_{x}\right) \\
& +\int_{\Gamma}\left(\eta u_{1} \cdot \overline{\sigma_{1}\left(w_{1, j}\right) \vec{n}_{1}}+\eta u_{2} \cdot \overline{\sigma_{2}\left(w_{2, j}\right) \vec{n}_{2}}-\sigma_{1}\left(\eta u_{1}\right) \vec{n}_{1} \cdot \overline{w_{1, j}}-\sigma_{2}\left(\eta u_{2}\right) \vec{n}_{2} \cdot \overline{w_{2, j}}\right) \mathrm{d} s_{x} .
\end{aligned}
$$

Exploiting the transmission conditions for $\left(w_{1, j}, w_{2, j}\right)$ on $\Gamma$ we obtain

$$
\begin{aligned}
\sum_{i=1}^{2} q_{i}\left(\eta u_{i}, w_{i, j}\right)= & \sum_{i=1}^{2}\left(\int_{\Omega_{i}} L_{i}\left(\eta u_{i}\right) \cdot \overline{w_{i, j}} \mathrm{~d} x+\int_{\Gamma_{i}} \eta u_{i} \cdot \overline{\sigma_{i}\left(w_{i, j}\right) \vec{n}_{i}} \mathrm{~d} s_{x}-\int_{\Gamma_{i}} \sigma_{i}\left(\eta u_{i}\right) \vec{n}_{i} \cdot \overline{w_{i, j}} \mathrm{~d} s_{x}\right) \\
& +\int_{\Gamma}\left(\eta\left(u_{1}-u_{2}\right) \cdot \overline{\sigma_{1}\left(w_{1, j}\right) \vec{n}_{1}}-\left(\sigma_{1}\left(\eta u_{1}\right) \vec{n}_{1}+\sigma_{2}\left(\eta u_{2}\right) \vec{n}_{2}\right) \cdot \overline{w_{1, j}}\right) \mathrm{d} s_{x} .
\end{aligned}
$$

Thus the assertion holds.

Remark 4.2. Theorem 4.4 is valid also in the case when $\Omega_{1} \cup \Omega_{2} \subset \operatorname{supp} \eta$, i.e. the cut-off function $\eta$ in (31) can be omitted

$$
c_{j}=\sum_{i=1}^{2}\left(\int_{\Omega_{i}} f_{i} \cdot \overline{w_{i, j}} \mathrm{~d} x+\int_{\Gamma_{i}}\left(u_{i} \cdot \overline{\sigma_{i}\left(w_{i, j}\right) \vec{n}_{i}}-\sigma_{i}\left(u_{i}\right) \vec{n}_{i} \cdot \overline{w_{i, j}}\right) \mathrm{d} s_{x}\right)+\int_{\Gamma}\left(p_{1} \cdot \overline{\sigma_{1}\left(w_{1, j}\right) \vec{n}_{1}}-p_{2} \cdot \overline{w_{1, j}}\right) \mathrm{d} s_{x} .
$$

The proof of Theorem 4.4 can be repeated in this case, taking into account that $w_{i, j}$ is smooth outside of a small vicinity of the corner point $P$.

Theorem 4.5. Suppose that the assumptions of Theorem 4.4 are satisfied and let $\left(W_{1, j}, W_{2, j}\right)$ be a solution of the two-dimensional transmission problem (7) with vanishing right-hand sides, which admits the decomposition

$$
\left(\begin{array}{l}
W_{1, j} \\
W_{2, j}
\end{array}\right)=\left(\begin{array}{l}
w_{1, j} \\
w_{2, j}
\end{array}\right)+\left(\begin{array}{c}
w_{1, j}^{\text {reg }} \\
w_{2, j}^{\text {reg }}
\end{array}\right)
$$

with $w_{i, j}^{\mathrm{reg}} \in\left[H^{1}\left(\Omega_{i}\right)\right]^{2}, i=1,2$. Then the following formula holds

$$
c_{j}=\sum_{i=1}^{2}\left(\int_{\Omega_{i}} f_{i} \cdot \overline{W_{i, j}} \mathrm{~d} x+\int_{\Gamma_{i}^{D}} g_{i} \cdot \overline{\sigma_{i}\left(W_{i, j}\right) \vec{n}_{i}} \mathrm{~d} s_{x}-\int_{\Gamma_{i}^{N}} h_{i} \cdot \overline{W_{i, j}} \mathrm{~d} s_{x}\right)+\int_{\Gamma}\left(p_{1} \cdot \overline{\sigma_{1}\left(W_{1, j}\right) \vec{n}_{1}}-p_{2} \cdot \overline{W_{1, j}}\right) \mathrm{d} s_{x} .
$$


Proof. Inserting the decomposition (33) into the two-dimensional problem (7) with vanishing right-hand sides we conclude that the functions $\left(w_{1, j}^{\mathrm{reg}}, w_{2, j}^{\mathrm{reg}}\right)$ satisfy the transmission problem

$$
\begin{aligned}
\mu_{i} \Delta w_{i, j}^{\mathrm{reg}}+\left(\lambda_{i}+\mu_{i}\right) \nabla \operatorname{div} w_{i, j}^{\text {reg }} & =0 & & \text { in } \Omega_{i}, \\
w_{i, j}^{\text {reg }} & =-w_{i, j} & & \text { on } \Gamma_{i}^{D}, \\
\sigma_{i}\left(w_{i, j}^{\mathrm{reg}}\right) \vec{n}_{i} & =-\sigma_{i}\left(w_{i, j}\right) \vec{n}_{i} & & \text { on } \Gamma_{i}^{N}, \\
w_{1, j}^{\mathrm{reg}}-w_{2, j}^{\text {reg }} & =-\left(w_{1, j}-w_{2, j}\right) & & \text { on } \Gamma, \\
\sigma_{1}\left(w_{1, j}^{\mathrm{reg}}\right) \vec{n}_{1}+\sigma_{2}\left(w_{2, j}^{\text {reg }}\right) \vec{n}_{2} & =-\left(\sigma_{1}\left(w_{1, j}\right) \vec{n}_{1}+\sigma_{2}\left(w_{2, j}\right) \vec{n}_{2}\right) & & \text { on } \Gamma .
\end{aligned}
$$

Since the right-hand sides of (35) vanish in the neighbourhood of the point $P$ and are smooth, the transmission problem (35) has a unique solution $\left(w_{1, j}^{\mathrm{reg}}, w_{2, j}^{\mathrm{reg}}\right)$ which belongs to $\left[H^{1}\left(\Omega_{1}\right)\right]^{2} \times\left[H^{1}\left(\Omega_{2}\right)\right]^{2}$. We have

$$
\sum_{i=1}^{2} q_{i}\left(u_{i}, W_{i, j}\right)=\sum_{i=1}^{2} q_{i}\left(u_{i}, w_{i, j}\right)+\sum_{i=1}^{2} q_{i}\left(u_{i}, w_{i, j}^{\mathrm{reg}}\right) .
$$

Because of Green's formula the expressions $q_{i}\left(u_{i}, w_{i, j}^{\text {reg }}\right), i=1,2$, vanish and we obtain

$$
\sum_{i=1}^{2} q_{i}\left(u_{i}, W_{i, j}\right)=\sum_{i=1}^{2} q_{i}\left(u_{i}, w_{i, j}\right)=c_{j}
$$

On the other hand

$$
\begin{aligned}
\sum_{i=1}^{2} q_{i}\left(u_{i}, W_{i, j}\right)= & \sum_{i=1}^{2}\left(\int_{\Omega_{i}} L_{i}\left(u_{i}\right) \cdot \overline{W_{i, j}} \mathrm{~d} x-\int_{\Omega_{i}} u_{i} \cdot \overline{L_{i}\left(W_{i, j}\right)} \mathrm{d} x \int_{\Gamma_{i}} u_{i} \cdot \overline{\sigma_{i}\left(W_{i, j}\right) \vec{n}_{i}} \mathrm{~d} s_{x}-\int_{\Gamma_{i}} \sigma_{i}\left(u_{i}\right) \vec{n}_{i} \cdot \overline{W_{i, j}} \mathrm{~d} s_{x}\right) \\
& +\int_{\Gamma}\left(u_{1} \cdot \overline{\sigma_{1}\left(W_{1, j}\right) \vec{n}_{1}}+u_{2} \cdot \overline{\sigma_{2}\left(W_{2, j}\right) \vec{n}_{2}}-\sigma_{1}\left(u_{1}\right) \vec{n}_{1} \cdot \overline{W_{1, j}}-\sigma_{2}\left(u_{2}\right) \vec{n}_{2} \cdot \overline{W_{2, j}}\right) \mathrm{d} s_{x} \\
= & \sum_{i=1}^{2}\left(\int_{\Omega_{i}} f_{i} \cdot \overline{W_{i, j}} \mathrm{~d} x+\int_{\Gamma_{i}^{D}} g_{i} \cdot \overline{\sigma_{i}\left(W_{i, j}\right) \vec{n}_{i}} \mathrm{~d} s_{x}-\int_{\Gamma_{i}^{N}} h_{i} \cdot \overline{W_{i, j}} \mathrm{~d} s_{x}\right) \\
& +\int_{\Gamma}\left(p_{1} \cdot \overline{\sigma_{1}\left(W_{1, j}\right) \vec{n}_{1}}-p_{2} \cdot \overline{W_{1, j}}\right) \mathrm{d} s_{x} .
\end{aligned}
$$

Thus (34) holds.

Let us compare the numerical effort due to the application of formulas (32) and (34). In order to evaluate (32) we have to compute the unknown boundary data $u_{i}$ or $\sigma_{i}\left(u_{i}\right) \vec{n}_{i}$ on $\Gamma_{i}$ for every loading. In order to apply formula (34) it is necessary to compute the unknown Cauchy data of the weight functions $\left(W_{1, j}, W_{2, j}\right)$ by solving the auxiliary transmission problem (35) and to calculate the weight function inside the domain $\Omega_{i}$ using a representation formula. Once computed for a specific domain, the weight functions $\left(W_{1, j}, W_{2, j}\right)$ can be used for the computation of stress intensity factors for different sets of loadings on this domain.

\section{Numerical approximation of the GSIF's}

\subsection{Error estimates for domain decomposition methods}

In order to apply formulae (32) or (34) to the numerical approximation of the coefficient $c_{j}$ in $(25)$ we have to solve the transmission problem (7) or (35), respectively. This is done by boundary element domain 
decomposition methods (see [31,33] for a detailed description of this method). We consider a variational formulation of the transmission problem (7). For the sake of simplicity we assume that zero body forces and homogeneous transmission conditions are given, i.e. $f_{1}=f_{2}=p_{1}=p_{2}=0$. Let us denote by $u_{i}$ the boundary displacements and by $t_{i}$ the corresponding boundary stresses on $\partial \Omega_{i}$. Let $\tilde{g}_{i} \in\left[H^{3 / 2}\left(\partial \Omega_{i}\right)\right]^{2}, i=1,2$, be extensions of the given Dirichlet data $g_{i}$ with $\tilde{g}_{1}=\tilde{g}_{2}$ on the interface $\Gamma$ and let $\tilde{h}_{i} \in\left[H^{1 / 2}\left(\partial \Omega_{i}\right)\right]^{2}, i=1,2$, be extensions of the given Neumann data $h_{i}$ with $\tilde{h}_{1}=\tilde{h}_{2}$ on $\Gamma$. We split the boundary data $u_{i}, t_{i}$ according to

$$
u_{i}=\tilde{u}_{i}+\tilde{g}_{i}, \quad t_{i}=\tilde{t}_{i}+\tilde{h}_{i} .
$$

Now we look for the functions $\left(\tilde{u}_{i}, \tilde{t}_{i}\right)$ in the Hilbert space $V$ defined by

$$
\begin{aligned}
V= & \left\{\left(\left(u_{1}, u_{2}\right),\left(t_{1}, t_{2}\right)\right) \in\left[\tilde{H}^{1 / 2}\left(\Gamma_{1}^{N} \cup \Gamma\right)\right]^{2} \times \tilde{[} H^{1 / 2}\left(\Gamma_{2}^{N} \cup \Gamma\right)\right]^{2} \times \tilde{H}^{-1 / 2}\left[\left(\Gamma_{1}^{D} \cup \Gamma\right)\right]^{2} \times \\
& \left.\tilde{H}^{-1 / 2}\left[\left(\Gamma_{2}^{D} \cup \Gamma\right)\right]^{2} ; u_{1}=u_{2} \text { on } \Gamma\right\} .
\end{aligned}
$$

Furthermore, we introduce for real $s$ the space

$$
H^{s}=\left[H^{s}\left(\partial \Omega_{1}\right)\right]^{2} \times\left[H^{s}\left(\partial \Omega_{2}\right)\right]^{2} \times\left[H^{s-1}\left(\partial \Omega_{1}\right)\right]^{2} \times\left[H^{s-1}\left(\partial \Omega_{2}\right)\right]^{2}
$$

with the norm

$$
\left\|\left(\left(u_{1}, u_{2}\right),\left(t_{1}, t_{2}\right)\right)\right\|_{H^{s}}^{2}=\sum_{i=1}^{2}\left\{\left\|u_{i}\right\|_{\left[H^{s}\left(\partial \Omega_{i}\right)\right]^{2}}^{2}+\left\|t_{i}\right\|_{\left[H^{s-1}\left(\partial \Omega_{i}\right)\right]^{2}}^{2}\right\}
$$

Note that $V$ is a subspace of $H^{1 / 2}$.

The "symmetric" variational formulation of the transmission problem for $(\tilde{u}, \tilde{t}) \operatorname{reads}$ as $[11,31,33]$ :

Find $\left(\left(\tilde{u}_{1}, \tilde{u}_{2}\right),\left(\tilde{t}_{1}, \tilde{t}_{2}\right)\right) \in V$ such that

$$
a(\tilde{u}, \tilde{t} ; \hat{u}, \hat{t})=-a(\tilde{g}, \tilde{h} ; \hat{u}, \hat{t}) \quad \forall(\hat{u}, \hat{t}) \in V
$$

where the bilinear form $a(\cdot, \cdot): V \times V \rightarrow \mathbb{R}$ is defined by

$$
a(\tilde{u}, \tilde{t} ; \hat{u}, \hat{t})=\sum_{i=1}^{2}\left\{\left\langle D_{i} \tilde{u}_{i}+1 / 2 \tilde{t}_{i}+K_{i}^{\prime} \tilde{t}_{i}, \hat{u}_{i}\right\rangle_{\partial \Omega_{i}}+\left\langle V_{i} \tilde{t}_{i}-1 / 2 \tilde{u}_{i}-K_{i} \tilde{u}_{i}, \hat{t}_{i}\right\rangle_{\partial \Omega_{i}}\right\}
$$

Here, $V_{i}$ is the single layer, $D_{i}$ the hyper-singular, $K_{i}$ the double layer and $K_{i}^{\prime}$ the adjoint double layer operator. The bilinear form $a(\cdot, \cdot)$ is continuous and positive definite on $V \times V$ provided that $\operatorname{meas}\left(\Gamma_{1}^{D} \cup \Gamma_{2}^{D}\right)>0[11]$. Otherwise we have to replace the space $V$ by $V_{N}=V / \mathcal{R}$, where $\mathcal{R}$ is the space of rigid motions and we have to assume that $\sum_{i=1}^{2} \int_{\Gamma_{i}} h_{i} r d s_{x}=0$ for all $r \in \mathcal{R}$

We remark that the right-hand side of (36) can be written as dual pairing on $V^{\prime} \times V$ using the Riesz isomorphism.

We denote by $S^{1}\left(\partial \Omega_{i}\right)$ the space of piecewise linear and by $S^{0}\left(\partial \Omega_{i}\right)$ the space of piecewise constant splines on $\partial \Omega_{i}$ with the mesh parameter $h$. Let $\left(\tilde{u}_{h}, \tilde{t}_{h}\right)=\left(\left(\tilde{u}_{h, 1}, \tilde{u}_{h, 2}\right),\left(\tilde{t}_{h, 1}, \tilde{t}_{h, 2}\right)\right)$ be the Galerkin solution of $(36)$ in the space

$$
V_{h}:=\left\{\left[S^{1}\left(\partial \Omega_{1}\right)\right]^{2} \times\left[S^{1}\left(\partial \Omega_{2}\right)\right]^{2} \times\left[S^{0}\left(\partial \Omega_{1}\right)\right]^{2} \times\left[S^{0}\left(\partial \Omega_{2}\right)\right]^{2}\right\} \cap V .
$$

We define the approximations $u_{h, i}, t_{h, i}$ of $u_{i}, t_{i}$ by

$$
u_{h, i}=\tilde{u}_{h, i}+\tilde{g}_{i}, \quad t_{h, i}=\tilde{t}_{h, i}+\tilde{h}_{i} .
$$


The errors of the approximation of $(u, t)$ and $(\tilde{u}, \tilde{t})$ are the same since

$$
u_{i}-u_{h, i}=\tilde{u}_{i}-\tilde{u}_{h, i}, \quad t_{i}-t_{h, i}=\tilde{t}_{i}-\tilde{t}_{h, i}
$$

The order of convergence depends on the regularity of the weak solution $(\tilde{u}, \tilde{t})$. Let $\alpha=\min$ Re $\alpha_{1}$, where the minimum is taken over all singular points $P$ and let $g_{i} \in\left[H^{3 / 2}\left(\Gamma_{i}^{D}\right)\right]^{2}, h_{i} \in\left[\tilde{H}^{1 / 2}\left(\Gamma_{i}^{N}\right)\right]^{2}$. From Theorem 4.1 follows that $u_{i}, \tilde{u}_{i} \in\left[H^{1 / 2+\alpha-\varepsilon}\left(\partial \Omega_{i}\right)\right]^{2}$ and $t_{i}, \tilde{t}_{i} \in\left[H^{-1 / 2+\alpha-\varepsilon}\left(\partial \Omega_{i}\right)\right]^{2}$ for a small positive $\varepsilon, i . e .(u, t),(\tilde{u}, \tilde{t}) \in$ $H^{1 / 2+\alpha-\varepsilon}$.

Lemma 5.1. Let $\tilde{g}_{i} \in\left[H^{3 / 2}\left(\partial \Omega_{i}\right)\right]^{2}, \tilde{h}_{i} \in\left[H^{1 / 2}\left(\partial \Omega_{i}\right)\right]^{2}$ be fixed extensions of the given Dirichlet and Neumann data and let $\tau, \beta$ be real numbers with $1 / 2-\alpha+\varepsilon \leq \tau \leq 1 / 2 \leq \beta \leq 1 / 2+\alpha-\varepsilon$. Then the Galerkin procedure converges asymptotically as

$$
\left\|(u, t)-\left(u_{h}, t_{h}\right)\right\|_{H^{\tau}} \leq d h^{\beta-\tau}\|(\tilde{u}, \tilde{t})\|_{H^{\beta}} .
$$

Proof. We follow the ideas of Hsiao/Wendland [10] where error estimates for boundary element methods are given under the assumption that the solution is smooth enough. Similar results to FEM discretization of problems with non smooth solutions were obtained in $[3,16]$.

We have

$$
\left\|(u, t)-\left(u_{h}, t_{h}\right)\right\|_{H^{\tau}}=\left\|(\tilde{u}, \tilde{t})-\left(\tilde{u}_{h}, \tilde{t}_{h}\right)\right\|_{H^{\tau}}=\sup _{\|(\zeta, \xi)\|_{H^{-\tau}=1}}\left|\left\langle\left(\tilde{u}-\tilde{u}_{h}, \tilde{t}-\tilde{t}_{h}\right),(\zeta, \xi)\right\rangle\right| .
$$

Since $(\zeta, \xi) \in H^{-\tau} \subset V^{\prime}$, there exist a unique solution $(\eta, \nu) \in H^{-\tau+1} \cap V$ of the adjoint variational problem

$$
a^{\prime}(\eta, \nu ; \hat{u}, \hat{t})=a(\hat{u}, \hat{t} ; \eta, \nu)=\langle(\hat{u}, \hat{t}),(\zeta, \xi)\rangle \quad \forall(\hat{u}, \hat{t}) \in V
$$

Note that the adjoint bilinear form $a^{\prime}(\cdot, \cdot): V \times V \rightarrow \mathbb{R}$ is continuous and positive definite and that the regularity results for (36) are also applicable to $(39)$. Let $\left(\eta_{h}, \nu_{h}\right) \in V_{h}$ be the Galerkin solution of (39). Then we obtain

$$
\begin{aligned}
\left\|(u, t)-\left(u_{h}, t_{h}\right)\right\|_{H^{\tau}} & =\sup _{\|(\zeta, \xi)\|_{H^{-\tau}}=1}\left|a\left(\tilde{u}-\tilde{u}_{h}, \tilde{t}-\tilde{t}_{h} ; \eta, \nu\right)\right| \\
& =\sup _{\|(\zeta, \xi)\|_{H^{-\tau}=1}}\left|a\left(\tilde{u}-\tilde{u}_{h}, \tilde{t}-\tilde{t}_{h} ; \eta-\eta_{h}, \nu-\nu_{h}\right)\right| .
\end{aligned}
$$

The continuity of the bilinear form $a(\cdot, \cdot)$ provides now

$$
\left\|(u, t)-\left(u_{h}, t_{h}\right)\right\|_{H^{\tau}} \leq d\left\|\left(\tilde{u}-\tilde{u}_{h}, \tilde{t}-\tilde{t}_{h}\right)\right\|_{H^{1 / 2}} \sup _{\|(\zeta, \xi)\|_{H^{-\tau}}=1}\left\|\left(\eta-\eta_{h}, \nu-\nu_{h}\right)\right\|_{H^{1 / 2}} .
$$

From error estimates in the energy norm for solutions on boundary element domain decomposition methods [9,11] we obtain finally

$$
\left\|(u, t)-\left(u_{h}, t_{h}\right)\right\|_{H^{\tau}} \leq d h^{\beta-1 / 2}\|(\tilde{u}, \tilde{t})\|_{H^{\beta}} \cdot h^{-\tau+1 / 2} \sup _{\|(\zeta, \xi)\|_{H^{-\tau}=1}}\|(\eta, \nu)\|_{H^{-\tau+1}} .
$$

The assertion follows now from the a priori estimate

$$
\|(\eta, \nu)\|_{H^{-\tau+1}} \leq k\|(\zeta, \xi)\|_{H^{-\tau}}
$$

with a positive constant $k$, since $\|(\zeta, \xi)\|_{H^{-\tau}}=1$. 


\subsection{Error estimates for the GSIF's}

Let us apply formula (34) to the numerical approximation of the coefficient $c_{j}$ in (25). To this end we have to compute the unknown boundary data of the regular part $w_{i, j}^{\text {reg }}$ of the weight function $W_{i j}$ by solving the transmission problem (35). In the following we denote the computed Dirichlet data of $w_{i j}^{\mathrm{reg}}$ on $\partial \Omega_{i}$ by $\left(u_{1, j}^{h}, u_{2, j}^{h}\right)$ and the computed Neumann data on $\partial \Omega_{i}$ by $\left(t_{1, j}^{h}, t_{2, j}^{h}\right)$. Let $\tilde{g}_{i} \in\left[H^{3 / 2}\left(\partial \Omega_{i}\right)\right]^{2}, \tilde{h}_{i} \in\left[H^{1 / 2}\left(\partial \Omega_{i}\right)\right]^{2}$ be fixed extensions of the given Dirichlet and Neumann data. Since only homogeneous transmission data are given and $\left.W_{i, j}\right|_{\Gamma_{i}^{D}}=0,\left.\sigma_{i}\left(W_{i j}\right) \vec{n}_{i}\right|_{\Gamma_{i}^{N}}=0$, we can write formula (34) in the form

$$
c_{j}=\sum_{i=1}^{2}\left(\int_{\partial \Omega_{i}} \tilde{g}_{i} \cdot \overline{\sigma_{i}\left(W_{i, j}\right) \vec{n}_{i}} \mathrm{~d} s_{x}-\int_{\partial \Omega_{i}} \tilde{h}_{i} \cdot \overline{W_{i, j}} \mathrm{~d} s_{x}\right) .
$$

Inserting $W_{i, j}^{h}=w_{i, j}+u_{i, j}^{h}$ and $\sigma_{i}\left(W_{i, j}^{h}\right) \vec{n}_{i}=\sigma_{i}\left(w_{i, j}\right) \vec{n}_{i}+t_{i, j}^{h}$ into (40) we obtain the approximation $c_{j}^{h}$ of the coefficient $c_{j}$

$$
c_{j}^{h}=\sum_{i=1}^{2}\left(\int_{\partial \Omega_{i}} \tilde{g}_{i} \cdot \overline{\left(\sigma_{i}\left(w_{i, j}\right) \vec{n}_{i}+t_{i, j}^{h}\right)} \mathrm{d} s_{x}-\int_{\partial \Omega_{i}} \tilde{h}_{i} \cdot \overline{\left(w_{i, j}+u_{i, j}^{h}\right)} \mathrm{d} s_{x}\right) .
$$

The following error estimate is valid:

Lemma 5.2. Let $\tilde{g}_{i} \in\left[H^{3 / 2}\left(\partial \Omega_{i}\right)\right]^{2}, \tilde{h}_{i} \in\left[H^{1 / 2}\left(\partial \Omega_{i}\right)\right]^{2}$ be fixed extensions of the given Dirichlet and Neumann data and let $c_{j}^{h}$ be the approximation of the coefficient $c_{j}$ defined by (41). Then the following error estimate is valid

$$
\left|c_{j}-c_{j}^{h}\right| \leq d h^{2(\alpha-\varepsilon)}
$$

with a positive real constant $d$ and a small positive $\varepsilon$.

Proof. We have

$$
\left|c_{j}-c_{j}^{h}\right| \leq \sum_{i=1}^{2}\left(\left|\int_{\partial \Omega_{i}} \tilde{g}_{i} \cdot \overline{\left(\sigma_{i}\left(w_{i, j}^{\mathrm{reg}}\right) \vec{n}_{i}-t_{i, j}^{h}\right)} \mathrm{d} s_{x}\right|+\left|\int_{\partial \Omega_{i}} \tilde{h}_{i} \cdot \overline{\left(w_{i, j}^{\mathrm{reg}}-u_{i, j}^{h}\right)} \mathrm{d} s_{x}\right|\right) .
$$

Note that according to Theorem $4.1 w_{i, j}^{\text {reg }} \in\left[H^{1 / 2+\alpha-\varepsilon}\left(\partial \Omega_{i}\right)\right]^{2}, \sigma_{i}\left(w_{i, j}^{\text {reg }}\right) \vec{n}_{i} \in\left[H^{-1 / 2+\alpha-\varepsilon}\left(\partial \Omega_{i}\right)\right]^{2}$. Since $\tilde{g}_{i} \in$ $\left[H^{3 / 2}\left(\partial \Omega_{i}\right)\right]^{2}$ and $\tilde{h}_{i} \in\left[H^{1 / 2}\left(\partial \Omega_{i}\right)\right]^{2}$ we can estimate

$$
\begin{aligned}
\left|c_{j}-c_{j}^{h}\right| \leq & \sum_{i=1}^{2}\left(\left\|\tilde{g}_{i}\right\|_{\left[H^{1 / 2+\alpha-\varepsilon}\left(\partial \Omega_{i}\right)\right]^{2}}\left\|\sigma_{i}\left(w_{i, j}^{\mathrm{reg}}\right) \vec{n}_{i}-t_{i, j}^{h}\right\|_{\left[H^{-1 / 2-\alpha+\varepsilon}\left(\partial \Omega_{i}\right)\right]^{2}}\right. \\
& \left.+\left\|\tilde{h}_{i}\right\|_{\left[H^{-1 / 2+\alpha-\varepsilon}\left(\partial \Omega_{i}\right)\right]^{2}}\left\|w_{i, j}^{\mathrm{reg}}-u_{i, j}^{h}\right\|_{\left[H^{1 / 2-\alpha+\varepsilon}\left(\partial \Omega_{i}\right)\right]^{2}}\right) .
\end{aligned}
$$

Lemma 5.1 with $\tau=1 / 2-\alpha+\varepsilon$ and $\beta=1 / 2+\alpha-\varepsilon$ provides us the assertion.

Remark. Similar estimates were obtained in [3] for the FEM approximation of the SIF's.

Example. We consider the simple boundary value problem

$$
\begin{aligned}
\Delta u & =0 & & \text { in } \Omega, \\
u & =g:=r^{\alpha} \sin (\alpha \omega) & & \text { on } \partial \Omega
\end{aligned}
$$




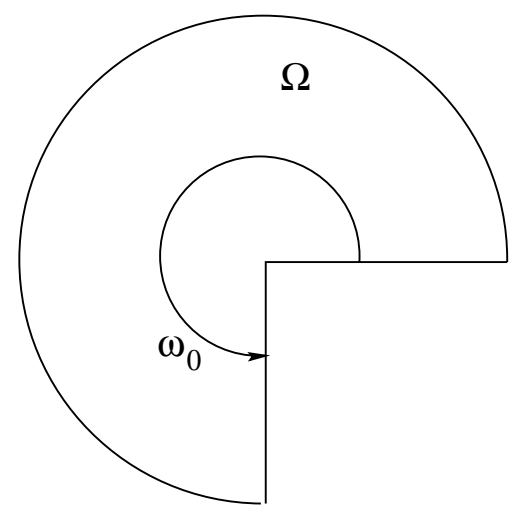

TABLE 1.

\begin{tabular}{|c||c|}
\hline $\mathrm{N}$ & $\left|c-c_{h}\right|$ \\
\hline \hline 28 & 0.024589 \\
\hline 56 & 0.008667 \\
\hline 112 & 0.003687 \\
\hline 224 & 0.001720 \\
\hline 448 & 0.000836 \\
\hline
\end{tabular}

FiguRE 4. A bounded sector.

in a bounded sector with the opening angle $\omega_{0}$ (see Fig. 4).

In this case the solution of the boundary value problem coincides with the given Dirichlet data and we have $\alpha=\pi / \omega_{0}$. The stress intensity factor is equal to 1 and can be calculated by a special case of formula (34)

$$
c=\int_{\partial \Omega} g \partial_{n} w \mathrm{~d} s_{x}
$$

where

$$
w=-\frac{1}{\pi} r^{-\alpha} \sin (\alpha \omega)+w^{\mathrm{reg}}
$$

and $w^{\text {reg }} \in H^{1 / 2+\alpha-\varepsilon}(\partial \Omega)$ is a solution of (44) with $g=(1 / \pi) r^{-\alpha} \sin (\alpha \omega)$. The theoretical rate of convergence according to the estimate $(42)$ is $h^{2(\alpha-\varepsilon)}$. In Figure 5 we compare the theoretical and the numerical rate of convergence in dependence on the singular exponent $\alpha \in(0.5,1.0)$ corresponding to the opening angle $\omega_{0} \in(\pi, 2 \pi)$. Here the numerical order of convergence is computed by

$$
\log \frac{\left|c-c_{h}^{192}\right|}{\left|c-c_{h}^{382}\right|}
$$

where $c_{h}^{m}$ denotes the approximation of the coefficient $c$ using a uniform mesh with $m$ elements. One should remark that for small $m$ the numerical rate of convergence is better then the theoretical one, but the agreement between the theoretical and numerical values improves for increasing $m$.

In order to demonstrate the absolute accuracy of the approximation we show in the Table 1 the relative error of the approximation in the dependence on the number of boundary elements. Here we take $\alpha=0.5008$ i.e. $\omega_{0} \sim 2 \pi$.

\subsection{The Motz' anti-plane crack problem}

In Section 4 we have restricted our considerations to the main part of the asymptotics, i.e. we have considered only singular exponents $\alpha_{j}$ with $0<\operatorname{Re} \alpha_{j}<1$. This considerations can be easily extended to exponents of higher order, provided that the boundary is straight in the neighbourhood of the corner points and the given data are smooth enough. Otherwise it would be necessary to calculate the explicit influence of the geometry and of the given data on the asymptotics. In the following example we compute for a simple problem the stress intensity factors of higher order for which comparable results are available. 


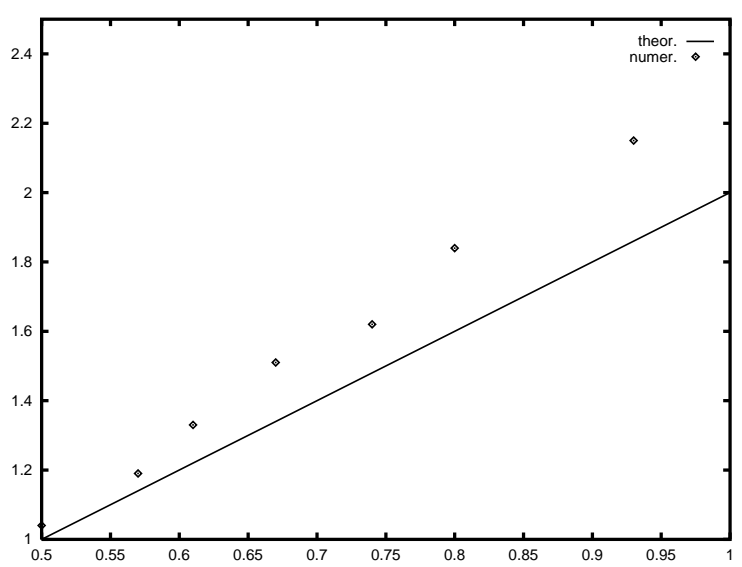

FiguRE 5. Theoretical and numerical approximation rate.

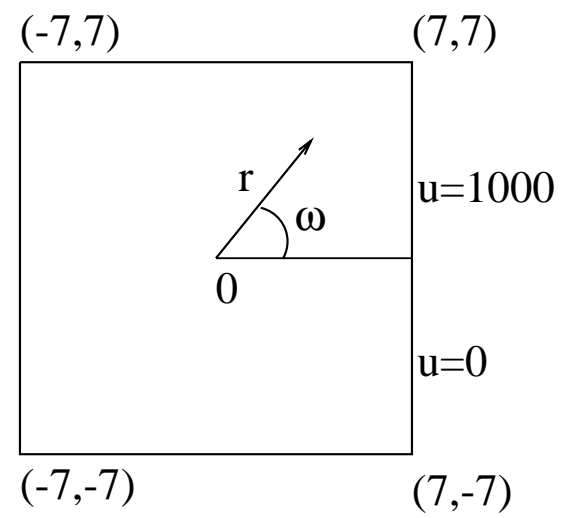

Figure 6. The Motz problem.

Let $\Omega$ be a square with corners in the points $C=(-7,7), B=(7,7), E=(-7,-7)$ and $F=(7,-7)$ containing a slit or a crack as shown in Figure 6. The Motz' anti-plane crack problem requires the solution of the Laplace equation

$$
\Delta u=0 \text { in } \Omega
$$

with boundary conditions

$$
\begin{array}{ll}
u=0 & \text { on } \overline{F G}, \\
u=1000 & \text { on } \overline{A B}
\end{array}
$$

and

$$
\partial_{n} u=0
$$


TABLE 2.

\begin{tabular}{|c||c|c|c|}
\hline \multicolumn{1}{|c||}{ Techniques used } & $\alpha_{1}$ & $\alpha_{2}$ & $\alpha_{3}$ \\
\hline \hline $\begin{array}{c}\text { Conformal Transformation Method } \\
\text { (Rosser/Papamichael [31]) }\end{array}$ & 151.625 & 4.733 & 0.133 \\
\hline $\begin{array}{c}\text { Boundary Integral Equation Method } \\
\text { (Xanthis } \text { et al. } \text { [40]) }\end{array}$ & 151.63 & 4.73 & 0.133 \\
\hline $\begin{array}{c}\text { Singularity Subtraction Technique } \\
\text { plus Local Mesh Refinement } \\
\text { (Liu/Lee/Pan [20]) }\end{array}$ & 151.634 & 4.729 & 0.134 \\
\hline $\begin{array}{c}\text { Motz' Technique plus } \\
\text { Local Mesh Refinement }\end{array}$ & & & \\
(Liu/Lee/Pan [20]) & 151.698 & 4.759 & 0.141 \\
\hline $\begin{array}{c}\text { Weight Functions Method } \\
\text { (Bochniak/Sändig) }\end{array}$ & & & \\
$\mathrm{N}=38$ & 152.28748 & 4.7388568 & 0.11565881 \\
$\mathrm{~N}=78$ & 152.10532 & 4.7621737 & 0.12983250 \\
$\mathrm{~N}=158$ & 151.89941 & 4.7535156 & 0.13264147 \\
$\mathrm{~N}=318$ & 151.77058 & 4.7446978 & 0.13310424 \\
$\mathrm{~N}=638$ & 151.69987 & 4.7391951 & \\
$\mathrm{~N}=1278$ & 151.66297 & & \\
\hline \multicolumn{2}{|c|}{} & & \\
\hline
\end{tabular}

on the rest of the boundary including the slit. The solution of this boundary value problem has in the vicinity of the point 0 the representation

$$
u(r, \omega)=500+\sum_{k=1}^{\infty} \alpha_{k} r^{k-1 / 2} \cos (k-1 / 2) \omega
$$

In Table 2 we compare the results computed by using formula (34) with results obtained by several other methods $[18,27,37]$.

\subsection{Numerical example for a bimaterial joint}

Let us consider a simple coupled structure consisting of two different linear elastic materials (see Fig. 7). On the left-hand side of the structure Dirichlet conditions are given, whereas on the remaining part of the boundary Neumann conditions are prescribed.

The displacement fields $\left(u_{1}, u_{2}\right)$ have in the neighbourhood of the point $P_{2}$ the asymptotics

$$
u_{i} \sim c \cdot r^{\alpha} \varphi_{i}(\omega)+O(r)
$$

If the material constants

$$
E_{i}=\frac{\mu_{i}\left(3 \lambda_{i}+2 \mu_{i}\right)}{\lambda_{i}+\mu_{i}}, \quad \nu_{i}=\frac{\lambda_{i}}{2\left(\lambda_{i}+\mu_{i}\right)}
$$

are chosen as

$$
E_{1}=20000.0 \mathrm{MPa}, \quad E_{2}=200000.0 \mathrm{MPa}, \quad \nu_{1}=\nu_{2}=0.35,
$$

then $\alpha \approx 0.774147$. 


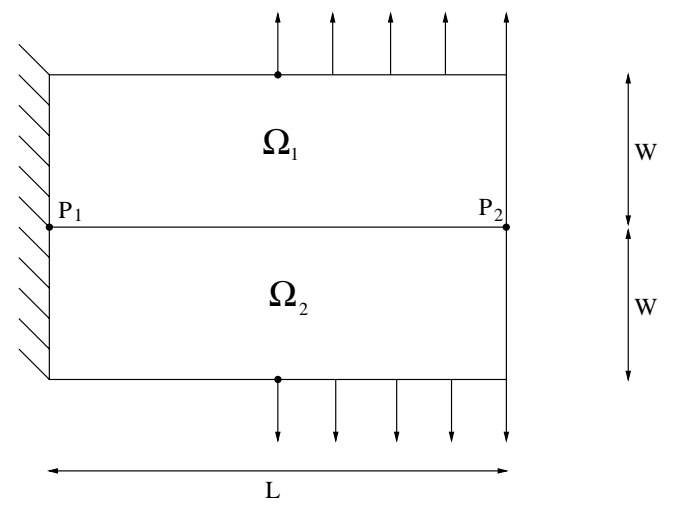

FiguRe 7. A bimaterial joint.



FiguRE 8. The main stress intensity factor $c$.

We calculate the leading stress intensity factor $c$ in (52) using formula (34). For the computation of the unknown Cauchy data of the solution $\left(u_{1}, u_{2}\right)$ on $\Gamma_{1} \cup \Gamma_{2}$ and of the weight functions $\left(W_{1}, W_{2}\right)$ we use the boundary element domain decomposition program 2DBEMDD developed by Steinbach [32]. The boundaries $\Gamma_{1}$ and $\Gamma_{2}$ are discretized by uniform meshes.

Let us investigate the dependence of the stress intensity factor $c$ in (52) on the width $W$. The applied force is here $f=2000 \mathrm{MPa}$, the length of the structure is fixed $L=1$. The material constants are chosen here as before: $\nu_{1}=\nu_{2}=0.35, E_{1}=20000 \mathrm{MPa}$. Figure 8 shows the leading stress intensity factor $c$ in dependence on the width $W$ for different values of the constant $E_{2}$.

The limit passage $W \rightarrow 0$ leads to a singular perturbed problem and the leading stress intensity factor $c$ seems to become infinite. 


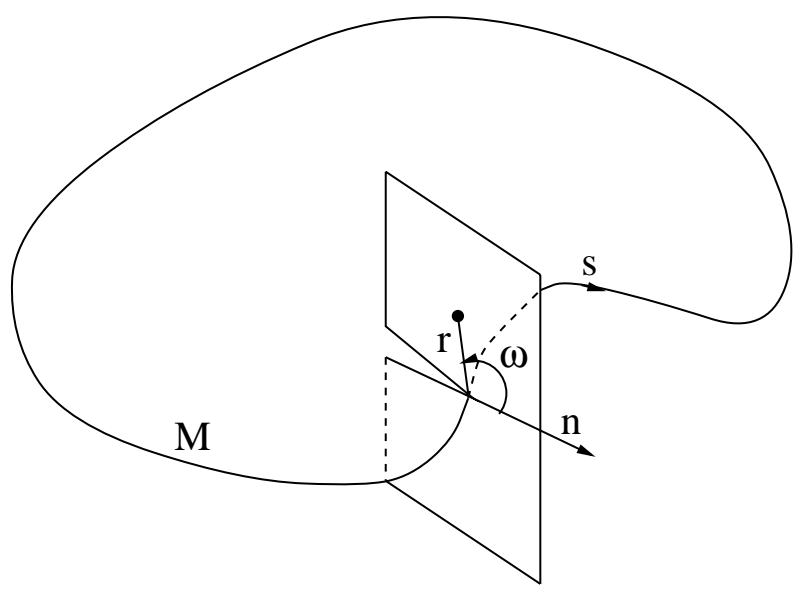

FiguRE 9. Plane interface crack.

\section{ThreE-Dimensional PLANE INTERFACE CRACKS}

\subsection{Asymptotic expansions}

Now we proceed to the 3D plane interface crack problem, assuming that the crack front $M \subset \partial \Gamma$ is smooth and that Neumann conditions are given on the crack surfaces. The results $[6,20,22,28]$ about asymptotic expansions for boundary value problems can be immediately extended to boundary transmission problem using ideas from [24].

Let us calculate the explicit asymptotics of the displacement field in the neighbourhood of a plane interface crack following the general theory of elliptic problems in domain with edges (see Chaps. 11, 12 in [23]). It is convenient to formulate the asymptotic expansions in local curvilinear coordinates $(r, \omega, s)$ defined by

$$
\left(\begin{array}{l}
x \\
y \\
z
\end{array}\right)=\left(\begin{array}{c}
\gamma_{1}(s) \\
\gamma_{2}(s) \\
0
\end{array}\right)+r \cos \omega\left(\begin{array}{c}
-\gamma_{2}^{\prime}(s) \\
\gamma_{1}^{\prime}(s) \\
0
\end{array}\right)+r \sin \omega\left(\begin{array}{l}
0 \\
0 \\
1
\end{array}\right)
$$

where $\left[0, L_{M}\right) \ni s \mapsto\left(\gamma_{1}(s), \gamma_{2}(s), 0\right)$ is a parametrisation of the crack front $M$ with respect to the arc length $s$ (see Figs. 2, 9).

If we denote by $u_{i}^{r}, u_{i}^{\omega}, u_{i}^{s}$ the components of the displacement vector $u_{i}$ corresponding to the coordinates $(r, \omega, s)$, then we obtain a decomposition of the Lamé operator $L_{i}$ and of the linearized stress tensor $\sigma_{i}(u)$ in a toroidal neighbourhood of the crack front $M$

$$
\begin{aligned}
L_{i} & =L_{i}^{0}+\tilde{L}_{i}, \\
\sigma_{i} & =\sigma_{i}^{0}+\tilde{\sigma}_{i},
\end{aligned}
$$

with

$$
L_{i}^{0} u=L_{i}^{0}\left(u_{i}^{r}, u_{i}^{\omega}, u_{i}^{s}\right)=\left(\begin{array}{r}
\left(\lambda_{i}+2 \mu_{i}\right) \frac{\partial}{\partial r}\left[\frac{\partial u_{i}^{r}}{\partial r}+\frac{1}{r} \frac{\partial u_{i}^{\omega}}{\partial \omega}+\frac{1}{r} u_{i}^{r}\right]-\mu_{i} \frac{1}{r} \frac{\partial}{\partial \omega}\left[\frac{\partial u_{i}^{\omega}}{\partial r}-\frac{1}{r} \frac{\partial u_{i}^{r}}{\partial \omega}+\frac{1}{r} u_{i}^{\omega}\right] \\
\left(\lambda_{i}+2 \mu_{i}\right) \frac{1}{r} \frac{\partial}{\partial \omega}\left[\frac{\partial u_{i}^{r}}{\partial r}+\frac{1}{r} \frac{\partial u_{i}^{\omega}}{\partial \omega}+\frac{1}{r} u_{i}^{r}\right]+\mu_{i} \frac{\partial}{\partial r}\left[\frac{\partial u_{i}^{\omega}}{\partial r}-\frac{1}{r} \frac{\partial u_{i}^{r}}{\partial \omega}+\frac{1}{r} u_{i}^{\omega}\right] \\
\mu_{i}\left[\frac{\partial^{2} u_{i}^{s}}{\partial r^{2}}+\frac{1}{r} \frac{\partial u_{i}^{s}}{\partial r}+\frac{1}{r^{2}} \frac{\partial^{2} u_{i}^{s}}{\partial \omega^{2}}\right]
\end{array}\right)
$$


and

$$
\begin{aligned}
\sigma_{i}^{0}\left(u_{i}\right)^{r r} & =2 \mu_{i} r \partial_{r} u_{i}^{r}+\lambda_{i}\left[r \partial_{r} u_{i}^{r}+\partial_{\omega} u_{i}^{\omega}+u_{i}^{r}\right], \\
\sigma_{i}^{0}\left(u_{i}\right)^{\omega \omega} & =2 \mu_{i}\left[\partial_{\omega} u_{i}^{\omega}+u_{i}^{r}\right]+\lambda_{i}\left[\partial_{\omega} u_{i}^{\omega}+r \partial_{r} u_{i}^{r}+u_{i}^{r}\right] \\
\sigma_{i}^{0}\left(u_{i}\right)^{s s} & =\lambda_{i}\left[r \partial_{r} u_{i}^{r}+u_{i}^{r}+\partial_{\omega} u_{i}^{\omega}\right], \\
\sigma_{i}^{0}\left(u_{i}\right)^{r \omega} & =\mu_{i}\left[r \partial_{r} u_{i}^{\omega}-u_{i}^{\omega}+\partial_{\omega} u_{i}^{r}\right], \\
\sigma_{i}^{0}\left(u_{i}\right)^{r s} & =\mu_{i} r \partial_{r} u_{i}^{s}, \\
\sigma_{i}^{0}\left(u_{i}\right)^{\omega s} & =\mu_{i} \partial_{\omega} u_{i}^{s} .
\end{aligned}
$$

For functions $v(r, \omega, s)$ with $v \sim O\left(r^{\alpha}\right)$ we have

$$
\begin{array}{ll}
L_{i}^{0} v \sim O\left(r^{\alpha-2}\right), & \tilde{L}_{i} v \sim O\left(r^{\alpha-1}\right), \\
\sigma_{i}^{0} v \sim O\left(r^{\alpha-1}\right), & \tilde{\sigma}_{i} v \sim O\left(r^{\alpha}\right) .
\end{array}
$$

The main part of the asymptotics of the three-dimensional displacement field $\left(u_{1}, u_{2}\right)$ in the neighbourhood of the smooth crack front $M$ is determined only by the principal parts of the operators $L_{i}$ and $\sigma_{i}(u)$ with coefficients frozen pointwise in $M$, i.e. by the operators $L_{i}^{0}$ and $\sigma_{i}^{0}(u)$. Thus the asymptotics of the displacement field $u_{i}=\left(u_{i}^{r}, u_{i}^{\omega}, u_{i}^{s}\right)$ in $\Omega_{i}$ has the form

$$
\left(\begin{array}{c}
u_{i}^{r} \\
u_{i}^{\omega} \\
u_{i}^{s}
\end{array}\right)(r, \omega, s) \sim c_{1}(s) r^{1 / 2+i \varepsilon}\left(\begin{array}{c}
\varphi_{i, 1}^{r}(\omega) \\
\varphi_{i, 1}^{\omega}(\omega) \\
0
\end{array}\right)+c_{2}(s) r^{1 / 2-i \varepsilon}\left(\begin{array}{c}
\varphi_{i, 2}^{r}(\omega) \\
\varphi_{i, 2}^{\omega}(\omega) \\
0
\end{array}\right)+c_{3}(s) r^{1 / 2}\left(\begin{array}{c}
0 \\
0 \\
\varphi_{i, 3}^{s}(\omega)
\end{array}\right)+O(r),
$$

with $\varepsilon$ defined by (11). Here, $1 / 2$ is an eigenvalue of the operator pencil $\mathcal{A}_{\Delta}$ with the eigenfunction $\left(\varphi_{1,3}^{s}, \varphi_{2,3}^{s}\right)$ and $1 / 2 \pm i \varepsilon$ are eigenvalues of $\mathcal{A}_{L}$ with the eigenfunctions $\left(\left(\varphi_{1,1}^{r}, \varphi_{1,1}^{\omega}\right),\left(\varphi_{2,1}^{r}, \varphi_{2,1}^{\omega}\right)\right)$ and $\left(\left(\varphi_{1,2}^{r}, \varphi_{1,2}^{\omega}\right),\left(\varphi_{2,2}^{r}, \varphi_{2,2}^{\omega}\right)\right)$, respectively.

Analogously to elliptic boundary value problems in domains with edges [23] we have

Theorem 6.1. Let $f_{i} \in\left[L_{2}\left(\Omega_{i}\right)\right]^{3}, g_{i} \in\left[H^{3 / 2}\left(\Gamma_{i}^{D}\right)\right]^{3}, h_{i} \in\left[\tilde{H}^{1 / 2}\left(\Gamma_{i}^{N}\right)\right]^{3}, p_{1} \in\left[H^{3 / 2}(\Gamma)\right]^{3}, p_{2} \in\left[\tilde{H}^{1 / 2}(\Gamma)\right]^{3}$. Furthermore we assume that there exist functions $w_{i} \in\left[H^{2}\left(\Omega_{i}\right)\right]^{3}$ with

$$
\begin{aligned}
\left.w_{i}\right|_{\Gamma_{i}^{D}} & =\left.g_{i}\right|_{\Gamma_{i}^{D}}, \\
\left.\sigma_{i}\left(w_{i}\right) \vec{n}_{i}\right|_{\Gamma_{i}^{N}} & =\left.h_{i}\right|_{\Gamma_{i}^{N}}, \\
\left.\left(w_{1}-w_{2}\right)\right|_{\Gamma} & =\left.p_{1}\right|_{\Gamma}, \\
\left.\left(\sigma_{1}\left(w_{1}\right) \vec{n}_{1}+\sigma_{2}\left(w_{2}\right) \vec{n}_{2}\right)\right|_{\Gamma} & =\left.p_{2}\right|_{\Gamma} .
\end{aligned}
$$

Then the asymptotics (56) of the solution of the three-dimensional problem (7) is valid in the neighbourhood of the crack front $M$. Furthermore we have $c_{j}(\cdot) \in H^{1 / 2}(M)$.

\subsection{Coefficient formulas for stress intensity distributions}

For the computation of the stress intensity distributions $c_{i}(s)$ in $(56)$ it is convenient to approximate them by elements of an finite dimensional function space. Let $\left\{e_{1}, \ldots, e_{n}\right\}$ be a basis of a $n$-dimensional space of splines defined on the edge $M$, i.e. $e_{k}: M \rightarrow \mathbb{R}$. We approximate $c_{i}(s)$ by a linear combination

$$
c_{j}(s) \approx \sum_{k=1}^{n} c_{j, k} \cdot e_{k}(s)
$$


This leads to a square system of linear equations for the unknown coefficients $c_{j, k}$

$$
\int_{M} c_{j}(s) \cdot \overline{e_{l}(s)} \mathrm{d} s=\sum_{k=1}^{n} c_{j, k} \cdot\left(\int_{M} e_{k}(s) \cdot \overline{e_{l}(s)} \mathrm{d} s\right)
$$

with a positive definite matrix. The moments

$$
\int_{M} c_{j}(s) \cdot \overline{e_{l}(s)} \mathrm{d} s
$$

of the coefficients $c_{j}(s)$ can be calculated using similar ideas as in Section 4. To this end we write the asymptotics (56) for the displacement fields $\left(u_{1}, u_{2}\right)$ in $\Omega_{1} \cup \Omega_{2}$ in the form

$$
\left(\begin{array}{l}
u_{1} \\
u_{2}
\end{array}\right)(r, \omega, s) \sim c_{1}(s) r^{\alpha_{1}}\left(\begin{array}{c}
\Phi_{1,1}(\omega) \\
\Phi_{2,1}(\omega)
\end{array}\right)+c_{2}(s) r^{\alpha_{2}}\left(\begin{array}{c}
\Phi_{1,2}(\omega) \\
\Phi_{2,2}(\omega)
\end{array}\right)+c_{3}(s) r^{\alpha_{3}}\left(\begin{array}{c}
\Phi_{1,3}(\omega) \\
\Phi_{2,3}(\omega)
\end{array}\right)
$$

and introduce the functions

$$
\Psi_{i, 1}(\omega)=\left(\begin{array}{c}
\psi_{i, 1}^{r} \\
\psi_{i, 1}^{\omega} \\
0
\end{array}\right), \quad \Psi_{i, 2}(\omega)=\left(\begin{array}{c}
\psi_{i, 2}^{r} \\
\psi_{i, 2}^{\omega} \\
0
\end{array}\right), \quad \Psi_{i, 3}(\omega)=\left(\begin{array}{c}
0 \\
0 \\
\psi_{i, 3}^{s}
\end{array}\right),
$$

where the vector functions $\left(\left(\psi_{1,1}^{r}, \psi_{1,1}^{\omega}\right),\left(\psi_{2,1}^{r}, \psi_{2,1}^{\omega}\right)\right)$ and $\left(\left(\psi_{1,2}^{r}, \psi_{1,2}^{\omega}\right),\left(\psi_{2,2}^{r}, \psi_{2,2}^{\omega}\right)\right)$ are eigensolutions of the operator pencil $\mathcal{A}_{L}$ corresponding to the eigenvalues $-\bar{\alpha}_{1,2}=-1 / 2 \pm i \varepsilon$, respectively, and the vector function $\left(\psi_{1,3}^{s}, \psi_{2,3}^{s}\right)$ is an eigensolution of $\mathcal{A}_{\Delta}$ corresponding to the eigenvalue $-\bar{\alpha}_{3}=-1 / 2$. According to (54) the operator $L_{i}^{0}$ can be decomposed into the two-dimensional Lamé operator acting on the displacement components $u_{i}^{r}, u_{i}^{\omega}$ and the two-dimensional Laplace operator acting on the displacement component $u_{i}^{s}$. Thus we can use Lemmata 4.2 and 4.3 in order to biorthonormalize the functions $\Phi_{i, j}$ and $\Psi_{i, j}$. From Lemma 4.3 it follows that for a fixed choice of $\left(\Phi_{1, j}, \Phi_{2, j}\right), j=1,2,3$, the functions $\left(\Psi_{1, j}, \Psi_{2, j}\right), j=1,2,3$, can be chosen in such a way that the following biorthonormality condition is satisfied

$$
\left.\sum_{i=1}^{2} \int_{\omega_{i-1}}^{\omega_{i}}\left(\sigma_{i}^{0}\left(r^{\alpha_{l}} \Phi_{i, l}(\omega)\right) \vec{n}_{i} \cdot \overline{r^{-\overline{\alpha_{k}}} \Psi_{i, k}(\omega)}-r^{\alpha_{l}} \Phi_{i, l}(\omega) \cdot \overline{\sigma_{i}^{0}\left(r^{-\bar{\alpha}_{k}} \Psi_{i, k}(\omega)\right) \vec{n}_{i}}\right)\right|_{r=\delta} \delta \mathrm{d} \omega=\delta_{l k}
$$

for $l, k=1,2,3$ and $\delta>0$.

Theorem 6.2. Suppose that the assumptions of Theorem 6.1 are satisfied. Let $h \in C^{2}(M)$ and let $\eta \in C_{0}^{\infty}\left(\mathbb{R}_{0}^{+}\right)$ be a cut-off function with $\eta(r)=1$ for $0 \leq r \leq \delta$. We define the functions

$$
\left(\begin{array}{l}
\Psi_{1, j}^{h} \\
\Psi_{2, j}^{h}
\end{array}\right)=\eta(r) r^{-\bar{\alpha}_{j}} h(s)\left(\begin{array}{c}
\Psi_{1, j} \\
\Psi_{2, j}
\end{array}\right)(\omega), \quad j=1,2,3 .
$$

Then the following integral formula holds

$$
\begin{aligned}
\int_{M} c_{j}(s) \cdot \overline{h(s)} \mathrm{d} s= & \sum_{i=1}^{2}\left(\int_{\Omega_{i}} f_{i} \cdot \overline{\Psi_{i, j}^{h}} \mathrm{~d} x-\int_{\Omega_{i}} u_{i} \cdot \overline{L_{i}\left(\Psi_{i, j}^{h}\right)} \mathrm{d} x+\int_{\Gamma_{i}}\left(u_{i} \cdot \overline{\sigma_{i}\left(\Psi_{i, j}^{h}\right) \vec{n}_{i}}-\sigma_{i}\left(u_{i}\right) \vec{n}_{i} \cdot \overline{\Psi_{i, j}^{h}}\right) \mathrm{d} s_{x}\right) \\
& +\int_{\Gamma}\left(p_{1} \cdot \overline{\sigma_{1}\left(\Psi_{1, j}^{h}\right) \vec{n}_{1}}-p_{2} \cdot \overline{\Psi_{1, j}^{h}}\right) \mathrm{d} s_{x} .
\end{aligned}
$$


Proof. We insert the solutions $u_{i}$ of the three-dimensional problem (7) and the functions $\Psi_{i, j}^{h}$ into Green's formula in $\Omega_{i} \backslash T_{\delta}$, where the torus $T_{\delta}$ is defined by

$$
T_{\delta}=\left\{(r, \omega, s) \in \mathbb{R}^{3}: 0 \leq r \leq \delta, 0 \leq \omega \leq 2 \pi, s \in\left[0, L_{M}\right)\right\} .
$$

Exploiting the transmission conditions for $\left(u_{1}, u_{2}\right)$ and $\left(\Psi_{1, j}^{h}, \Psi_{2, j}^{h}\right)$ on $\Gamma$ we obtain

$$
\begin{aligned}
& \sum_{i=1}^{2}\left(\int_{\Omega_{i} \backslash T_{\delta}} f_{i} \cdot \overline{\Psi_{i, j}^{h}} \mathrm{~d} x-\int_{\Omega_{i} \backslash T_{\delta}} u_{i} \cdot \overline{L_{i}\left(\Psi_{i, j}^{h}\right)} \mathrm{d} x+\int_{\Gamma_{i} \backslash T_{\delta}}\left(u_{i} \cdot \overline{\sigma_{i}\left(\Psi_{i, j}^{h}\right) \vec{n}_{i}}-\sigma_{i}\left(u_{i}\right) \vec{n}_{i} \cdot \overline{\Psi_{i, j}^{h}}\right) \mathrm{d} s_{x}\right) \\
& +\int_{\Gamma \backslash T_{\delta}}\left(p_{1} \cdot \overline{\sigma_{1}\left(\Psi_{1, j}^{h}\right) \vec{n}_{1}}-p_{2} \cdot \overline{\Psi_{1, j}^{h}}\right) \mathrm{d} s_{x}=\sum_{i=1}^{2} \int_{\partial T_{\delta} \cap \Omega_{i}}\left(\sigma_{i}\left(u_{i}\right) \vec{n}_{i} \cdot \overline{\Psi_{i, j}^{h}}-u_{i} \cdot \overline{\sigma_{i}\left(\Psi_{i, j}^{h}\right) \vec{n}_{i}}\right) \mathrm{d} s_{x} .
\end{aligned}
$$

We calculate the limit of the right-hand side of (59) as $\delta \rightarrow 0$. To this end we insert the asymptotics (56) of $u_{i}$ as $r=\delta \rightarrow 0$ into (59). Further, because of (55) we can replace the stress tensor $\sigma_{i}\left(u_{i}\right)$ by $\sigma_{i}^{0}\left(u_{i}\right)$. Taking into account that the area element $\mathrm{d} s_{x}$ on $T_{\delta}$ is given by

$$
\mathrm{d} s_{x}=\delta(1-\delta \cos (\omega) k(s)) \mathrm{d} \omega \mathrm{d} s,
$$

where $k(s)$ denotes the curvature of the crack front $M$ at the point with the arclength $s$, we obtain

$$
\begin{aligned}
& \lim _{\delta \rightarrow 0} \sum_{i=1}^{2} \int_{\partial T_{\delta} \cap \Omega_{i}}\left(\sigma_{i}\left(u_{i}\right) \vec{n}_{i} \cdot \overline{\Psi_{i, j}^{h}}-u_{i} \cdot \overline{\sigma_{i}\left(\Psi_{i, j}^{h}\right) \vec{n}_{i}}\right) \mathrm{d} s_{x} \\
& =\lim _{\delta \rightarrow 0} \sum_{i=1}^{2} \int_{\partial T_{\delta} \cap \Omega_{i}}\left(\sigma_{i}^{0}\left(\sum_{k=1}^{3} c_{k}(s) r^{\alpha_{k}} \Phi_{i, k}+O(r)\right) \vec{n}_{i} \cdot \overline{h(s) r^{-\bar{\alpha}_{j}} \Psi_{i, j}}\right. \\
& \left.-\left(\sum_{k=1}^{3} c_{k}(s) r^{\alpha_{k}} \Phi_{i, k}+O(r)\right) \cdot \overline{\sigma_{i}^{0}\left(h(s) r^{-\bar{\alpha}_{j}} \Psi_{i, j}\right) \vec{n}_{i}}\right)\left.\right|_{r=\delta} \mathrm{d} s_{x} \\
& =\lim _{\delta \rightarrow 0} \sum_{i=1}^{2} \int_{M} \sum_{k=1}^{3} c_{k}(s) \overline{h(s)} \int_{\omega_{i-1}}^{\omega_{i}}\left(\sigma_{i}^{0}\left(r^{\alpha_{k}} \Phi_{i, k}(\omega)\right) \vec{n}_{i} \cdot \overline{r^{-\overline{\alpha_{j}}} \Psi_{i, j}(\omega)}\right. \\
& \left.-r^{\alpha_{k}} \Phi_{i, k}(\omega) \cdot \overline{\sigma_{i}^{0}\left(r^{-\bar{\alpha}_{j}} \Psi_{i, j}(\omega)\right) \vec{n}_{i}}\right)\left.\right|_{r=\delta} \delta(1-\delta \cos (\omega) k(s)) \mathrm{d} \omega \mathrm{d} s \\
& =\sum_{i=1}^{2} \int_{M} \sum_{k=1}^{3} c_{k}(s) \cdot \overline{h(s)} \delta_{k, j} \mathrm{~d} s=\int_{M} c_{j}(s) \cdot \overline{h(s)} \mathrm{d} s .
\end{aligned}
$$

Here we have used the biorthonormality condition (57) and the fact that $\sigma_{0}$ is linear with respect to functions depending only on the variable $s$ i.e. $\sigma_{0}(c(s) u(r, \omega))=c(s) \sigma_{0}(u(r, \omega))$.

Theorem 6.3. Suppose that the assumptions of Theorem 6.1 are satisfied. Let $\left(W_{1, j}^{h}, W_{2, j}^{h}\right)$ be a solution of the three-dimensional transmission problem (7) with vanishing right-hand sides, which admits a decomposition

$$
\left(\begin{array}{l}
W_{1, j}^{h} \\
W_{2, j}^{h}
\end{array}\right)=\left(\begin{array}{c}
\Psi_{1, j}^{h} \\
\Psi_{2, j}^{h}
\end{array}\right)+\left(\begin{array}{c}
w_{1, j}^{\text {reg }} \\
w_{2, j}^{\text {reg }}
\end{array}\right), \quad j=1,2,3
$$


with $w_{i, j}^{\mathrm{reg}} \in\left[H^{1}\left(\Omega_{i}\right)\right]^{3}, i=1,2$. Then the following formula holds

$$
\begin{aligned}
\int_{M} c_{j}(s) \cdot \overline{h(s)} \mathrm{d} s= & \sum_{i=1}^{2}\left(\int_{\Omega_{i}} f_{i} \cdot \overline{W_{i, j}^{h}} \mathrm{~d} x+\int_{\Gamma_{i}^{D}} g_{i} \cdot \overline{\sigma_{i}\left(W_{i, j}^{h}\right) \vec{n}_{i}} \mathrm{~d} s_{x}-\int_{\Gamma_{i}^{N}} h_{i} \cdot \overline{W_{i, j}^{h}} \mathrm{~d} s_{x}\right) \\
& +\int_{\Gamma}\left(p_{1} \cdot \overline{\sigma_{1}\left(W_{1, j}^{h}\right) \vec{n}_{1}}-p_{2} \cdot \overline{W_{1, j}^{h}}\right) \mathrm{d} s_{x} .
\end{aligned}
$$

Proof. Inserting the decomposition (60) into the three-dimensional problem (7) with vanishing right-hand sides we conclude that the functions $\left(w_{1, j}^{\mathrm{reg}}, w_{2, j}^{\text {reg }}\right)$ satisfy the transmission problem

$$
\begin{aligned}
L_{i}\left(w_{i, j}^{\mathrm{reg}}\right) & =-L_{i}\left(\Psi_{i, j}^{h}\right) & & \text { in } \Omega_{i}, \\
w_{i, j}^{\text {reg }} & =-\Psi_{i, j}^{h} & & \text { on } \Gamma_{i}^{D}, \\
\sigma_{i}\left(w_{i, j}^{\mathrm{reg}}\right) \vec{n}_{i} & =-\sigma_{i}\left(\Psi_{i, j}^{h}\right) \vec{n}_{i} & & \text { on } \Gamma_{i}^{N}, \\
w_{1, j}^{\mathrm{reg}}-w_{2, j}^{\mathrm{reg}} & =-\left(\Psi_{1, j}^{h}-\Psi_{2, j}^{h}\right) & & \text { on } \Gamma, \\
\sigma_{1}\left(w_{1, j}^{\mathrm{reg}}\right) \vec{n}_{1}+\sigma_{2}\left(w_{2, j}^{\mathrm{reg}}\right) \vec{n}_{2} & =-\left(\sigma_{1}\left(\Psi_{1, j}^{h}\right) \vec{n}_{1}+\sigma_{2}\left(\Psi_{2, j}^{h}\right) \vec{n}_{2}\right) & & \text { on } \Gamma .
\end{aligned}
$$

Because of (55) the right-hand sides of (62) are regular enough and the transmission problem (62) has a unique solution $\left(w_{1, j}^{\mathrm{reg}}, w_{2, j}^{\mathrm{reg}}\right)$ which belongs to $\left[H^{1}\left(\Omega_{1}\right)\right]^{3} \times\left[H^{1}\left(\Omega_{2}\right)\right]^{3}$. The proof of formula (61) is analogous to the proof of formula (58).

\section{REFERENCES}

[1] I. Babuška and A. Miller, The post-processing approach in the finite element method, Part 2: The calculation of stress intensity factors. Internat. J. Numer. Methods Engrg. 20 (1984) 1111-1129.

[2] I. Babuška, T. von Petersdorff and B. Andersson, Numerical treatment of vertex singularities and intensity factors for mixed boundary value problems for the Laplace equation in $R^{3}$. SIAM J. Numer. Anal. 31 (1994) 1265-1288.

[3] M. Bourlard, M. Dauge, M.-S. Lubuma and S. Nicaise, Coefficients of the singularities for elliptic boundary value problems on domains with conical points III: Finite element methods on polygonal domains. SIAM J. Numer. Anal. 29 (1992) $136-155$.

[4] H.F. Bueckner, A novel principle for the computation of stress intensity factors. ZAMM 50 (1970) 529-546.

[5] H.F. Bueckner, Weight functions and fundamental fields for the penny-shaped and the half-plane crack in three-space. Internat. J. Solids and Structures 23 (1987) 57-93.

[6] M. Costabel and M. Dauge, General edge asymptotics of solutions of second-order elliptic boundary value problems I, II. Proc. Roy. Soc. Edinburgh A 123 (1993) 109-155, 157-184.

[7] M. Costabel and M. Dauge, Computation of corner singularities in linear elasticity, in Boundary Value Problems and Integral Equations in Nonsmooth Domains, M. Costabel, M. Dauge, S. Nicaise Eds., Marcel Dekker Inc. (1995).

[8] M. Dobrowolski, Numerical Approximation of Elliptic Interface and Corner Problems. Habilitationsschrift, University of Bonn (1981).

[9] G.C. Hsiao, B.N. Khoromskij and W.L. Wendland, Boundary integral operators and domain decomposition. Preprint 94-11, Mathematisches Institut A, Universität Stuttgart (1994).

[10] G.C. Hsiao and W.L. Wendland, The Aubin-Nitsche Lemma for integral equations. J. Integral Equations 3 (1981) $299-315$.

[11] G.C. Hsiao and W.L. Wendland, Domain decomposition in boundary element methods, in Domain Decomposition Methods for Partial Differential Equations, R. Glowinski et al. Eds., SIAM (1991) 41-49.

[12] C. Hwu, C.J. Kao and L.E. Chang, Delamination fracture criteria for composite laminates. J. Composite Mat. 29 (1995) $1962-1987$.

[13] M.F. Kanninen and C.H. Popelar, Advanced Fracture Mechanics. Oxford University Press, New York (1985).

[14] S.N. Karp and F.C. Karal, The elastic-field behaviour in the neighbourhood of a crack of arbitrary angle. Comm. Pure Appl. Math. 15 (1962) 413-421.

[15] V.A. Kondrat'ev, Boundary problems for elliptic equations in domains with conical or angular points. Trans. Moscow Math. Soc. 16 (1967) 209-292.

[16] A. Kufner and A.-M. Sändig, Some Applications of Weighted Sobolev Spaces. Teubner, Leipzig (1987).

[17] M. Lenczner, Méthode de calcul du coefficient de singularité pour la solution du problème de Laplace dans un domaine diédral. Math. Modell. Numer. Anal. 27 (1993) 395-420. 
[18] K.M. Liu, K.M. Lee and C.K. Pan, Numerical techniques for determining stress intensity and higher order factors using the finite difference methods, in Computational Mechanics '95 Vol. 2, S.N. Atluri et al. Eds., Springer Verlag, Berlin (1995).

[19] V.G. Maz'ya and B.A. Plamenevsky, On the coefficients in the asymptotics of solutions of elliptic boundary value problems in domains with conical points. Math. Nachr. 76 (1977) 29-60.

[20] V.G. Maz'ya and J. Rossmann, Über die Asymptotik der Lösungen elliptischer Randwertaufgaben in der Umgebung von Kanten. Math. Nachr. 138 (1988) 27-53.

[21] S.A. Nazarov, Derivation of the variational inequality for small increase of mode-one crack. Mech. Solids 24 (1989) $145-152$.

[22] S.A. Nazarov and B.A. Plamenevsky, The Neumann problem for selfadjoint elliptic systems in a domain with piecewise-smooth boundary. Amer. Math. Soc. Transl. (2) 155 (1993) 169-206.

[23] S.A. Nazarov and B.A. Plamenevsky, Elliptic Problems in Domains with Piecewise Smooth Boundaries. Walter de Gruyter, Berlin (1994).

[24] S. Nicaise and A.-M. Sändig, General interface problems I, II. Math. Methods Appl. Sci. 17 (1994) 395-429, 431-450.

[25] S. Nicaise and A.-M. Sändig, Transmission problems for the Laplace and elasticity operators: Regularity and boundary integral formulation. Math. Models Methods Appl. Sci. (to appear).

[26] J.R. Rice, Some remarks on elastic crack-tip stress fields. Internat. J. Solids and Structures 8 (1972) 751-758.

[27] J.B. Rosser and N. Papamichael, A power series solution of a harmonic mixed boundary value problem. MRC Technical Summary Report number 1405 (1975).

[28] J. Rossmann and A.-M. Sändig, Formulas for the coefficients in the asymptotics of solutions of boundary value problems for second order systems near edges. ZAMM 76 Suppl. 4 (1996) 181-184.

[29] H. Schmitz, K. Volk and W. Wendland, Three-dimensional singularities of elastic fields near vertices. Numer. Methods Partial Differential Equations 9 (1993) 23-337.

[30] T.L. Shan and H.F. Bückner, The weight function theory for piecewise homogeneous isotropic notches in antiplane strain. $J$. Appl. Mech. 55 (1988) 596-603.

[31] O. Steinbach, Gebietszerlegungsmethoden mit Randintegralgleichungen und effiziente numerische Lösungsverfahren für gemischte Randwertprobleme. Dissertation, Universität Stuttgart (1996).

[32] O. Steinbach, On the realization of boundary element methods for mixed boundary value problems. Preprint 98/07, SFB 404, Universität Stuttgart (1998).

[33] O. Steinbach and W.L. Wendland, Domain decomposition and preconditioning techniques in boundary element methods, in Boundary Element Topics, W.L. Wendland Ed., Springer Verlag, Berlin (1997) 473-492.

[34] B.A. Szabo and Z. Yosibash, Numerical analysis of singularities in two dimensions, Part 2: Computation of generalized flux/stress intensity factors. Internat. J. Numer. Methods Engrg. 39 (1996) 409-434.

[35] M.L. Williams, Stress singularities resulting from various boundary conditions in angular corners of plates in extension. $J$. Appl. Mech. 19 (1952) 526-528.

[36] M.L. Williams, The stresses around a fault or crack in dissimilar media. Bull. Seismol. Soc. Amer. 49 (1959) $199-204$.

[37] L.S. Xanthis, M.J.M. Bernal and C. Atkinson, The treatment of singularities in the calculation of stress intensity factors using the boundary integral equation method. Comput. Methods Appl. Mech. Engrg. 26 (1981) 285-304. 